\title{
Origin of Mafic Microgranular Enclaves (MMEs) and Their Host Rocks of the Cretaceous Xiaojiang-Liangnong Granitic Complexes in the Southeast Coast Magmatic Belt, S China
}

\author{
Pei-Shan Hsieh, Cheng-Hong Chen ${ }^{*}$, Chiung-Mei Yen, and Chi-Yu Lee \\ Department of Geosciences, National Taiwan University, Taipei, Taiwan, ROC
}

Received 31 March 2008, accepted 1 July 2008

\begin{abstract}
Cretaceous Late Yanshanian (LY) magmatism produced large amounts of I-type granitoids in the Southeast Coast Magmatic Belt (SCMB). I-type granitoids from Xiaojiang-Liangnong complexes (N Zhejiang) in the northern part of this belt are characterized by containing abundant mafic microgranular enclaves (MMEs). On the basis of petrography and mineralogy, two types of MMEs are recognized. One (Type 1), enclosed in the granodiorite, is composed of amphibole, high-Ti tabular biotite, plagioclase, K-feldspar, and quartz. Using the Al-in-amphibole geobarometer, a shallow origin $(4-8 \mathrm{~km})$ of amphiboles is suggested. The other (Type 2), enclosed in alkali feldspar granite, is amphibole-free and composed of low-Ti acicular biotite, plagioclase, K-feldspar, and quartz. Geochemistry of major and trace elements seems to reflect two different evolving trends for these host granitoids. The Sr-Nd isotopic features indicate that all the studied samples vary in a narrow range of $\operatorname{Isr}(0.7078$ to 0.7082$)$ and $\varepsilon \mathrm{Nd}_{(\mathrm{T})}(-5.6$ to -8.3$)$, except the Type 2 enclaves and hosts that show slightly more depleted compositions ( $\mathrm{Isr}=0.7073$ to 0.7075 and $\varepsilon \mathrm{Nd}_{(\mathrm{T})}=-5.0$ to -6.0 ). U-Pb zircon ages obtained are $109.6 \pm 0.9 \mathrm{Ma}$ for the granodiorite and 113.5 $\pm 1.1 \mathrm{Ma}$ for the alkali feldspar granite, with 103.2 $\pm 0.9 \mathrm{Ma}$ for the Type $2 \mathrm{MMEs}$. Inherited ages of $117.0 \pm 2.0 \mathrm{Ma}$ and $119.8 \pm 1.1 \mathrm{Ma}$ seem to present in the former two samples. Similarities of mineral chemistry and isotopic composition among the MMEs-host pairs as well as the younger age of MMEs suggest that MMEs of Xiaojiang-Liangnong complexes most likely represent mixing/mingling products formed as a consequence of the intrusion of basaltic magma into the host granitoids under different cooling conditions. Judging from the presence of inherited zircon ages, the composition gap between high-Ti and low-Ti biotite in granodiorite and alkali feldspar granite, and lower zircon saturation temperatures for the host granitoids (calculated as $787^{\circ} \mathrm{C}$ and $763^{\circ} \mathrm{C}$, respectively, for these two rock types), origin of the LY I-type granitoids in the Xiaojiang-Liangnong complexes is related to partial melting of different sources, with the preceding alkali feldspar granite derived from more quartzofeldspathic and later granodiorite, from more mafic sources.
\end{abstract}

Key words: Mafic microgranular enclaves, Host granitoids, Zircon saturation temperature, Southeast coast magmatic belt

Citation: Hsieh, P. S., C. H. Chen, C. M. Yen, and C. Y. Lee, 2009: Origin of mafic microgranular enclaves (MMEs) and their host rocks of the Cretaceous Xiaojiang-Liangnong granitic complexes in the southeast coast magmatic belt, S China. Terr. Atmos. Ocean. Sci., 20, 481-500, doi: 10.3319/TAO. 2008.07.01.01(TT)

\section{INTRODUCTION}

There are two fundamental theories for the evolution of I-type granites, assimilation fractional crystallization (AFC) from a more mafic magma, which is basically derived from the mantle and partial melting of crustal materials. The former is a traditional one and adopted by many igneous petrologists with some means of modification during the course of AFC (e.g., Pitcher 1997). The latter is mainly advocated

\footnotetext{
* Corresponding author

E-mail: chench@ntu.edu.tw
}

by those who focused on the observation of the Paleozoic granites of the Lachlan Fold Belt (LFB), SE Australia (Chappell and White 1992; Chappell 1999). Investigations of LFB granites suggested that I-type rocks fall into two groups, formed at high and low magmatic temperatures (e.g., Chappell et al. 1987). The distinction is made on the absence or presence, respectively, of zircon with inherited ages in the more mafic rocks, and on the related differences in $\mathrm{Zr}$ variation patterns with $\mathrm{SiO}_{2}$ (Chappell et al. 1998). Studies on zircon saturation temperatures $\left(\mathrm{T}_{\mathrm{Zr}}\right)$, calculated 
from $\mathrm{Zr}$ concentration in zircon and bulk-rock compositions, also demonstrated that initial magma temperature at the source can be distinguished between the inheritancerich (mean $766^{\circ} \mathrm{C}$ ) and inheritance-poor (mean $837^{\circ} \mathrm{C}$ ) granitoids (Miller et al. 2003) and offered important insight into the thermal history of granitic bodies.

Cretaceous granitoids in the Southeast Coast Magmatic Belt (SCMB) belong to I-type category (Jahn et al. 1990; Chen et al. 2000). Typical examples are the Fuzhou and Zhangzhou igneous complexes in $\mathrm{N}$ and $\mathrm{S}$ Fujian, where the formation of more felsic member has previously been explained by fractional crystallization from mafic suites (Martin et al. 1994; Chen et al. 2000). However, based on the shallow intrusion $(5-7 \mathrm{~km})$ of these granitic rocks and their emplacement predating the A-type granites and rhyolites, Chen et al. $(2004,2008)$ suggested that they are more likely derived from crustal melting due to basaltic underplating at a large degree of lithospheric stretching. To further assess the granite petrogenesis in this area, new approaches need to be conducted, such as an examination of zircon U-Pb age or investigation on the mafic microgranular enclaves (MMEs). Since MMEs are rather common in calc-alkaline granitoids (Didier 1991), they may provide evidence of the role of mafic magmas in the evolution of calc-alkaline granitic magmas and carry important information interpreting the origin of batholiths (Barbarin 2005).

In the Xiaojiang-Liangnong granitic complexes of $\mathrm{N}$ Zhejiang, or the northern part of the SCMB (Fig. 1a), some MMEs and sporadic mafic dikes are observed. In this paper, we conduct mineral chemistry, whole-rock geochemistry (major and trace elements), and isotope analysis ( $\mathrm{Sr}$ and $\mathrm{Nd}$ ) for both the MMEs and their host rocks to better understand the characteristics and origin of these MMEs as well as the Cretaceous granitoids in the SCMB. Ages reported previously for the major granitic plutons in N Zhejiang were mainly the ${ }^{40} \mathrm{Ar}{ }^{39} \mathrm{Ar}$ biotite dates (Chen et al. 1991). In this study, zircon U-Pb ages were also conducted for the host rocks of these MMEs. This helps to know the emplacement time and zircon saturation condition of the granitic magma, and to constrain the petrogenesis of granitoids in $\mathrm{N}$ Zhejiang through the information of high or low magmatic temperatures.

\section{GEOLOGICAL BACKGROUND AND SAMPLING}

The SCMB, characterized by large amounts of the Late Yanshanian I-type granitoids (ca. 110 - $99 \mathrm{Ma}$ ), is a NNEtrending magmatic belt about $900 \mathrm{~km}$ long and $150 \mathrm{~km}$ wide in the coastal region of Zhejiang and Fujian provinces (Fig. 1a). In Fujian province, i.e., the southern part of the SCMB, ages and petrological, mineralogical, and geochemical characteristics of the Fuzhou and Zhangzhou granitic complexes have been extensively studied (Martin et al. 1994; Lai 1995; Lapierre et al. 1997; Chen and Jahn 1998; Chen et al. 2000, 2004). On the other hand, although Xiaojiang and Liangnong granitic complexes in N Zhejiang are considered the equivalents in the northern part of this belt, relevant data are scarce. Conventionally, four main lithologies in two series are recognized: the granodiorite-monzogranite association and the biotite granite-alkali feldspar granite association. Xiaojiang is mainly composed of alkali feldspar granite with small amounts of granodiorite, and Liangnong is composed of monzogranite, granodiorite, and biotite granite. Besides which, the igneous body of Longwangtang alkali feldspar granite to the south can be regarded as an extension of the Xiaojiang main pluton (Fig. 1b).

Relatively, enclaves are rare in Fujian, but rather common in Zhejiang I-type granitoids. Typical enclaves are basically found in the granodiorite of Xiaojiang and Liangnong, and in the alkali feldspar granite of Xiaojiang as well. The Beizhang monzogranitic pluton exposed between the Xiaojiang and Liangnong complexes also contains some enclaves (Chen et al. 2005). However, these enclaves occurred differently in size; those that appear in the granodiorite mostly range from several to less than twenty centimeters with a well rounded to elongated form (Fig. 2a), whereas those that

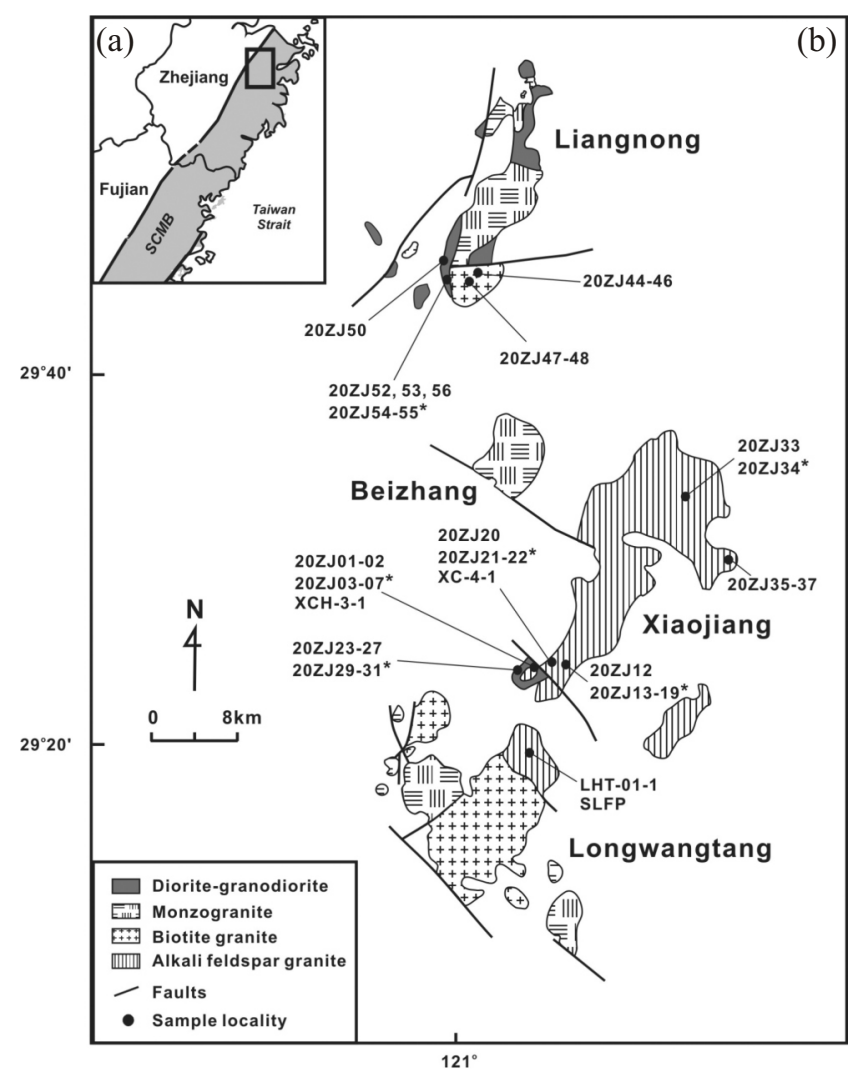

Fig. 1. (a) The Cretaceous Southeast Coast Magmatic Belt (SCMB) in the position of Zhejiang and Fujian provinces. (b) Geological map of the Xiaojiang-Liangnong igneous complexes and the adjacent Longwangtang igneous complex and Beizhang pluton in N Zhejinag (modified from BGMRZJ 1989). Sample numbers with a star denote enclaves of the granitoids. 
(a)

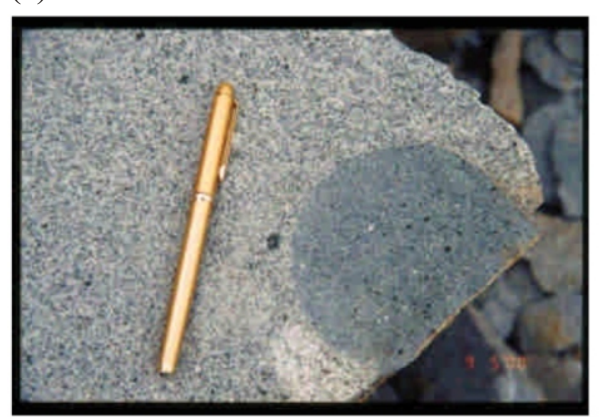

(c)

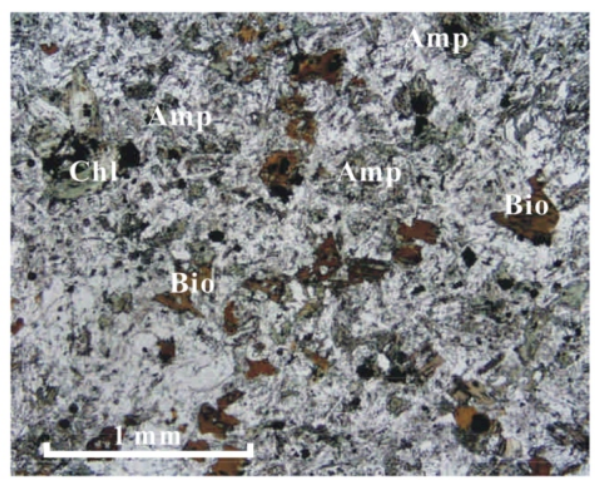

(b)

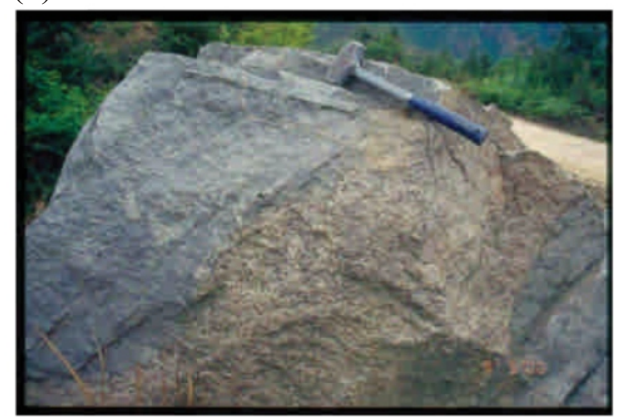

(d)

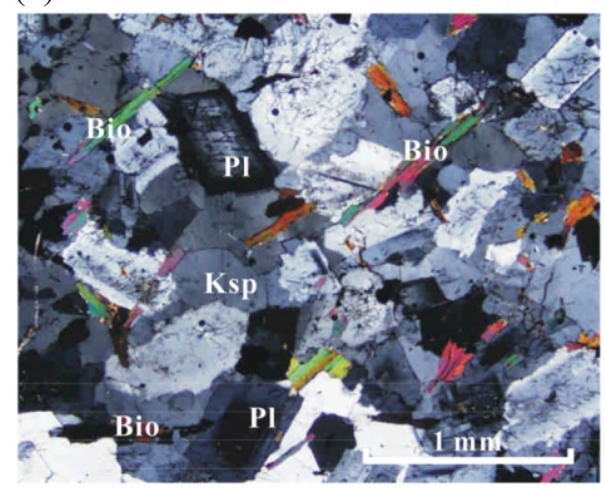

Fig. 2. Photographs of field occurrence of mafic microgranular enclaves (MMEs) in the Xiaojiang-Liangnong igneous complexes: (a) the Type 1 MMEs (darker portion) in the granodiorite host; and (b) the Type 2 MMEs (darker portion) in the alkali feldspar granite host, and photomicrographs of (c) Type 1 MMEs (20ZJ03) and (d) Type 2 MMEs (20ZJ12). Amp: amphibole; Bio: biotite; Chl: chlorite; Ksp: K-feldspar; and Pl: plagioclase.

appear in the alkali feldspar granite are generally greater than one meter (Fig. 2b). In some places, enclave swarms with acicular biotites in the latter type are observed. All these enclaves are richer in mafic minerals than their granitoid hosts, and thus can be grouped as the mafic microgranular enclaves (MMEs) (Didier 1991).

Lithology of these MMEs varies with their host rock associations. On the basis of mineralogy, two types of MMEs are classified: Type 1 for those containing evident hornblendes, enclosed in the hornblende-abundant granodiorite, and Type 2 for those that have no such mineral and appear in the hornblende-poor to hornblende-absent alkali feldspar granite. To envisage the relationship between the MMEs and their hosts, rocks enclosing these MMEs are the main target of this study (Fig. 1b). It is noted that rock samples for zircon $\mathrm{U}-\mathrm{Pb}$ dating are granodiorite and Type 2 MMEs from the Xiaojiang complex, and alkali feldspar granite from the Longwangtang complex.

\section{ANALYTICAL METHODS}

Mineral compositions were analyzed using a ShimadzuARL EMX-SM7 electron microprobe equipped with four channels of wavelength dispersive spectrometer (Department of Geosciences, National Taiwan University). The analyzing conditions were $15 \mathrm{KV}$ acceleration potential, 6 - $8 \mathrm{nA} \mathrm{sam}$ - ple current, and a beam spot $\sim 10 \mu \mathrm{m}$ in diameter. Data reduction is based on the ZAF correction procedure (Chen and Tung 1984). Amphibole analysis is particularly useful for the application of the $\mathrm{Al}$ in the hornblende geobarometer and zircon analysis, for the calculation of zircon saturation temperature. Data of the mineral composition (except zircon) have been listed separately (Yen 2005).

Discrete zircon grains were embayed in the epoxy resin and grained and polished to a surface suitable for U-Pb dating analysis. Cathodoluminescence (CL) images for zircons were obtained using a JOEL JSM-6360LV scanning electron microscope attached with a panchromatic CL imaging system (Gatan Mini-CL) at the Academia Sinica (Taipei), in order to characterize morphology and internal structures of zircons. U-Pb isotopic ratios and concentrations of zircons were determined on $40 \mu \mathrm{m}$ diameter regions of single zircons by laser ablation-inductively coupled plasma-mass spectrometry (LA-ICP-MS) at the Department of Geosciences, National Taiwan University, following Chiu et al. (2009). The ICP-MS used is an Agilent 7500s coupled to the UP213 laser ablation system (New Wave Research/Merchantek). ${ }^{207} \mathrm{~Pb} /{ }^{206} \mathrm{~Pb},{ }^{206} \mathrm{~Pb} /{ }^{238} \mathrm{U},{ }^{207} \mathrm{~Pb} /{ }^{235} \mathrm{U}$, and ${ }^{208} \mathrm{~Pb} /{ }^{232} \mathrm{Th}$ ratios calculated using GLITTER 4.0 software (Macquarie University) were corrected using GJ-1 (Jackson et al. 2004) as external standard. The reference Harvard zircon 91500 and Mud Tank (MT) zircon, used as unknown samples, 
yielded a weighted mean ${ }^{206} \mathrm{~Pb} /{ }^{238} \mathrm{U}$ age of $1062.1 \pm 1.7 \mathrm{Ma}$ $(\mathrm{n}=121)$ and $734.0 \pm 2.4 \mathrm{Ma}(\mathrm{n}=51)$, respectively, which are in good agreement with the recommended ages of $1061 \pm$ 4.0 Ma and $731 \pm 3.9 \mathrm{Ma}$ (Jackson et al. 2004). Measured compositions were carried out common-lead corrections using the procedure of Andersen (2002). All the reported ages and the concordia diagrams were made using ISOPLOT (rev.3) (Ludwig 2003). In this study, weighted mean ${ }^{206} \mathrm{~Pb} /{ }^{238} \mathrm{U}$ ages are adopted for concordant Phanerozoic zircons (Compston et al. 1992), because such ages are more precise than ${ }^{207} \mathrm{~Pb} /{ }^{235} \mathrm{U}$ and ${ }^{207} \mathrm{~Pb} /{ }^{206} \mathrm{~Pb}$ ages for zircons younger than $1 \mathrm{Ga}$ (Williams 1998; Cawood et al. 2003). In Table 1, the analytical dating results are given.

Major and trace element abundances of rock samples were determined at Department of Geosciences, National Taiwan University, by X-ray fluorescence spectrometer (Lee et al. 1997) and inductively coupled plasma-mass spectrometry (ICP-MS). The analytical precision and accuracy are generally better than $5 \%$ for most trace elements. $\mathrm{Sr}$ and $\mathrm{Nd}$ isotope compositions were measured using a Finnigan ${ }^{\circledR}$ MAT 262 mass spectrometer at the Department of Earth Sciences, National Cheng Kung University. The isotopic ratios were corrected for mass fractionation by normalizing to ${ }^{86} \mathrm{Sr} /{ }^{88} \mathrm{Sr}=0.1194$ and ${ }^{146} \mathrm{Nd} /{ }^{144} \mathrm{Nd}=0.7219$. Long-term laboratory measurements for SRM $987 \mathrm{Sr}$ and La Jolla Nd standards yield $0.710239 \pm 0.000012(\mathrm{n}=30)$ and 0.511847 $\pm 0.000012(\mathrm{n}=30)$, respectively. Selected whole rock geochemical and $\mathrm{Sr}-\mathrm{Nd}$ isotopic analyses are listed in Tables 2 and 3 , respectively.

\section{PETROGRAPHY AND MINERAL COMPOSITIONS}

The hornblende-abundant granodiorite hosts of the Type 1 MMEs consist of quartz ( $10-25 \mathrm{vol} \%)$, alkali feldspar (4 $17 \mathrm{vol} \%$ ), plagioclase (37 - $61 \mathrm{vol} \%)$, biotite (11 - $17 \mathrm{vol} \%$ ), hornblende (10 - $14 \mathrm{vol} \%)$, pyroxene (0.6 - $1.2 \mathrm{vol} \%)$ and Fe-Ti oxides ( $1-2$ vol\%). The hornblende-poor to hornblende-absent alkali feldspar granite hosts of the Type 2 MMEs consist of quartz (27 - 39 vol\%), alkali feldspar (33 $59 \mathrm{vol} \%)$, plagioclase (5 - $22 \mathrm{vol} \%)$, biotite $(1-10 \mathrm{vol} \%)$, hornblende ( $0-8$ vol\%), and accessory Fe-Ti oxides.

Type 1 dioritic MMEs consist of plagioclase (62 - $65 \mathrm{vol} \%$ ), hornblende (11 - $15 \mathrm{vol} \%)$, biotite (12 - 14 vol\%), quartz (2 $7 \mathrm{vol} \%$ ), and alkali feldspar ( $2-5 \mathrm{vol} \%$ ), with minor amounts of clinopyroxene and orthopyroxene (altogether 0 - $1.6 \mathrm{vol} \%$ ) (Fig. 2c). Accessory minerals are Fe-Ti oxides, sphene and apatite. It should be noted that one enclave sample (20ZJ31) enclosed in Xiaojiang granodiorite is quite different from typical Type 1 MMEs. It consists predominantly of orthopyroxene and clinopyroxene (Opx » $\mathrm{Cpx}$ and altogether 56 vol\%) and plagioclase (41 vol\%), with small amounts of biotite (2.6 vol\%) and Fe-Ti oxides (0.4 vol\%). This particular MMEs sample, Type 1a, is obviously gabbroic in composi- tion, and exhibits a reaction rim in the contact with the host rock. Type 2 monzogranitic MMEs contain euhedral plagioclase (32 - $42 \mathrm{vol} \%$ ), subhedral alkali feldspar (28 - $31 \mathrm{vol} \%$ ), interstitial quartz (22 - $26 \mathrm{vol} \%)$, and significantly needlelike biotite ( $8-11 \mathrm{vol} \%)$. Accessory minerals are sphene and Fe-Ti oxides. They differ from the Type 1 MMEs by lacking hornblende and pyroxene and the presence of needle-like, instead of tabular, biotite (Fig. 2d). Mineral assemblages in Type 1 MMEs-host pair are identical; however, the MMEs are generally fine-grained and richer in mafic minerals than their hosts. On the other hand, Type 2 MMEs are dominated by plagioclase and alkali feldspar, whereas their hosts by quartz and alkali feldspar. Because of the occurrence of enclave swarms with mafic dikes in the field and the hornblende-poor ( 0 - 5 vol\%) character in mineralogy, Beizhang MMEs and host rocks are classified into Type 2 as well.

As to the mineral chemistry, one striking feature is that the chemical compositions of constituents in the MMEs are similar to the same mineral in their host rocks. For example, An content of plagioclase and Or content of alkali feldspar in Type $1\left(\mathrm{An}_{58-15}\right.$ and $\left.\mathrm{Or}_{92-73}\right)$ and Type $2\left(\mathrm{An}_{30-12}\right.$ and $\left.\mathrm{Or}_{94-85}\right)$ MMEs generally fall into the range of that in Type $1\left(\mathrm{An}_{58-20}\right.$ and $\left.\mathrm{Or}_{91-69}\right)$ and Type $2\left(\mathrm{An}_{42-8}\right.$ and $\left.\mathrm{Or}_{88-20}\right)$ hosts, respectively. Type 1a gabbroic MMEs contain plagioclase $\left(\mathrm{An}_{85-47}\right)$ with no alkali feldspar (Yen 2005). Similarly, overlapping of hornblende and clinopyroxene compositions between Type 1 MMEs and their host rocks is also observed (Yen 2005). The amphiboles of Type 1 MMEs-host pair are calcic amphiboles (Leake 1978) with $(\mathrm{Ca}+\mathrm{Na}) \geq 2.05, \mathrm{Na}<0.46,(\mathrm{Na}+$ $\mathrm{K})<0.60$ and $\mathrm{Si}=6.9-7.3$ based on 23 oxygen, and characterized by having lower total $\mathrm{Al}$ contents $(<6.5 \mathrm{wt} \%)$ and medium $\mathrm{Mg}$ values $[\mathrm{Mg} /(\mathrm{Mg}+\mathrm{Fe})=0.60-0.85]$. Overall, they are comparable with the Fujian Cretaceous I-type granitoids (Fig. 3a). Using the geobarometer of Schmidt (1992), these amphiboles were formed at a lower pressure of $\sim 1-2.5 \mathrm{~kb}$, same as the emplacing condition of major igneous complexes in the south part of the SCMB (Chen et al. 2004).

There are no discernable compositional differences of constituent minerals in these two MMEs-host pairs. However, the high $\mathrm{Ti}\left(\mathrm{TiO}_{2}>4 \mathrm{wt} \%\right)$ and low $\mathrm{Ti}\left(\mathrm{TiO}_{2}<3.5 \mathrm{wt} \%\right)$ characters are well preserved in the tabular and acicular biotites in the Type 1 and Type 2 MMEs-hosts, respectively, although $\mathrm{Fe} /(\mathrm{Fe}+\mathrm{Mg})$ values of biotite from different MMEs-host pairs are indistinguishable (Fig. 3b). This phenomenon is somewhat distinct from the case of Fujian I-type granitoids, which reveal a composition gap of $\mathrm{Fe} /(\mathrm{Fe}+\mathrm{Mg})$ values, rather than $\mathrm{Ti}$ contents, for the granodiorite-monzogranite-syenogranite association and the alkali feldspar granites. We suggest that the biotite of Type 1 MMEs-host pair have crystallized under higher temperature condition than that of Type 2 pair due to higher content in Ti (Henry et al. 2005). The composition of representative zircons, which has been designated to calculate the $\mathrm{T}_{\mathrm{Zr}}$ for the magma, varies with $66.8-62.8 \mathrm{wt} \% \mathrm{ZrO}_{2}, 1.81-1.22 \mathrm{wt} \% \mathrm{HfO}_{2}$, and 
Table 1. Analytical results of LA-ICP-MS zircon U-Pb dating for Xiaojiang and Longwangtang complexes in the Southeast Coast Magmatic Belt.

\begin{tabular}{|c|c|c|c|c|c|c|c|c|}
\hline \multirow[b]{2}{*}{ Analysis } & \multicolumn{4}{|c|}{ Ratios (Common-Pb Corrected) } & \multicolumn{4}{|c|}{ Ages (Common-Pb Corrected, Ma) } \\
\hline & $\begin{array}{c}{ }^{207} \mathrm{~Pb} /{ }^{206} \mathrm{~Pb} \\
(1 \sigma)\end{array}$ & $\begin{array}{c}{ }^{207} \mathrm{~Pb} /{ }^{235} \mathrm{U} \\
(1 \sigma)\end{array}$ & $\begin{array}{c}{ }^{206} \mathrm{~Pb} /{ }^{238} \mathrm{U} \\
(1 \sigma)\end{array}$ & $\begin{array}{c}{ }^{208} \mathrm{~Pb} /{ }^{232} \mathrm{Th} \\
(1 \sigma)\end{array}$ & $\begin{array}{c}{ }^{207} \mathrm{~Pb} /{ }^{206} \mathrm{~Pb} \\
(1 \sigma)\end{array}$ & $\begin{array}{c}{ }^{207} \mathrm{~Pb} /{ }^{235} \mathrm{U} \\
(1 \sigma)\end{array}$ & $\begin{array}{c}{ }^{206} \mathrm{~Pb} /{ }^{238} \mathrm{U} \\
(1 \sigma)\end{array}$ & $\begin{array}{c}{ }^{208} \mathrm{~Pb} /{ }^{232} \mathrm{Th} \\
(1 \sigma)\end{array}$ \\
\hline \multicolumn{9}{|c|}{ Xiaojiang granodorite } \\
\hline XCH-3-1-01 & $0.05102(0.00042)$ & $0.12229(0.00197)$ & $0.01738(0.00018)$ & $0.00607(0.00007)$ & $242(21)$ & $117(2)$ & $111(1)$ & $122(1)$ \\
\hline $\mathrm{XCH}-3-1-03$ & $0.05127(0.00072)$ & $0.11595(0.00272)$ & $0.01640(0.00019)$ & $0.00553(0.00008)$ & $253(32)$ & $111(2)$ & $105(1)$ & $111(2)$ \\
\hline XCH-3-1-04 & $0.04639(0.00089)$ & $0.11156(0.00321)$ & $0.01744(0.00020)$ & $0.00580(0.00008)$ & $18(38)$ & $107(3)$ & $111(1)$ & $117(2)$ \\
\hline $\mathrm{XCH}-3-1-05$ & $0.04845(0.00036)$ & $0.11616(0.00172)$ & $0.01739(0.00017)$ & $0.00585(0.00007)$ & $121(17)$ & $112(2)$ & $111(1)$ & $118(1)$ \\
\hline $\mathrm{XCH}-3-1-08$ & $0.04753(0.00253)$ & $0.11322(0.00725)$ & $0.01728(0.00023)$ & $0.00549(0.00006)$ & $76(118)$ & $109(7)$ & $110(1)$ & $111(1)$ \\
\hline $\mathrm{XCH}-3-1-12$ & $0.05301(0.00226)$ & $0.12585(0.00726)$ & $0.01722(0.00030)$ & $0.00567(0.00012)$ & $329(94)$ & $120(7)$ & $110(2)$ & $114(2)$ \\
\hline $\mathrm{XCH}-3-1-14$ & $0.04671(0.00132)$ & $0.10875(0.00433)$ & $0.01689(0.00023)$ & $0.00552(0.00010)$ & $34(57)$ & $105(4)$ & $108(1)$ & $111(2)$ \\
\hline XCH-3-1-16 & $0.04871(0.00037)$ & $0.11249(0.00173)$ & $0.01675(0.00017)$ & $0.00546(0.00006)$ & $134(20)$ & $108(2)$ & $107(1)$ & $110(1)$ \\
\hline XCH-3-1-17 & $0.04971(0.00046)$ & $0.11719(0.00204)$ & $0.01710(0.00018)$ & $0.00557(0.00006)$ & $181(21)$ & $113(2)$ & $109(1)$ & $112(1)$ \\
\hline $\mathrm{XCH}-3-1-18$ & $0.05053(0.00163)$ & $0.11953(0.00523)$ & $0.01716(0.00023)$ & $0.00599(0.00011)$ & $219(73)$ & $115(5)$ & $110(1)$ & $121(2)$ \\
\hline XCH-3-1-19 & $0.04800(0.00355)$ & $0.10825(0.00948)$ & $0.01636(0.00027)$ & $0.00519(0.00007)$ & $99(160)$ & $104(9)$ & $105(2)$ & $105(1)$ \\
\hline $\mathrm{XCH}-3-1-20$ & $0.04905(0.00269)$ & $0.11204(0.00748)$ & $0.01656(0.00023)$ & $0.00524(0.00004)$ & $150(135)$ & $108(7)$ & $106(1)$ & $106(1)$ \\
\hline $\mathrm{XCH}-3-1-23$ & $0.04954(0.00202)$ & $0.11504(0.00639)$ & $0.01684(0.00029)$ & $0.00800(0.00022)$ & $173(100)$ & $111(6)$ & $108(2)$ & $161(4)$ \\
\hline $\mathrm{XCH}-3-1-25$ & $0.05305(0.00165)$ & $0.12016(0.00529)$ & $0.01634(0.00025)$ & $0.00592(0.00011)$ & $33(72)$ & $115(5)$ & $105(2)$ & $119(2)$ \\
\hline XCH-3-1-26 & $0.04608(0.00130)$ & $0.10966(0.00397)$ & $0.01726(0.00021)$ & $0.00557(0.00010)$ & $2(53)$ & $106(4)$ & $110(1)$ & $112(2)$ \\
\hline XCH-3-1-29 & $0.04622(0.00224)$ & $0.10814(0.00625)$ & $0.01697(0.00023)$ & $0.00541(0.00009)$ & $9(99)$ & $104(6)$ & $108(1)$ & $109(2)$ \\
\hline $\mathrm{XCH}-3-1-30$ & $0.05355(0.00138)$ & $0.12936(0.00471)$ & $0.01752(0.00022)$ & $0.00551(0.00009)$ & $352(63)$ & $124(4)$ & $112(1)$ & $111(2)$ \\
\hline $\mathrm{XCH}-3-1-33$ & $0.05293(0.00086)$ & $0.12472(0.00323)$ & $0.01709(0.00020)$ & $0.00546(0.00007)$ & $326(38)$ & $119(3)$ & $109(1)$ & $110(1)$ \\
\hline $\mathrm{XCH}-3-1-34$ & $0.04866(0.00046)$ & $0.11700(0.00207)$ & $0.01744(0.00018)$ & $0.00559(0.00007)$ & $131(22)$ & $112(2)$ & $111(1)$ & $113(1)$ \\
\hline $\mathrm{XCH}-3-1-35$ & $0.04801(0.00149)$ & $0.11540(0.00488)$ & $0.01743(0.00023)$ & $0.00566(0.00009)$ & $100(65)$ & $111(4)$ & $111(1)$ & $114(2)$ \\
\hline $\mathrm{XCH}-3-1-36$ & $0.05381(0.00426)$ & $0.13164(0.00130)$ & $0.01775(0.00040)$ & $0.00551(0.00013)$ & $363(170)$ & $126(12)$ & $113(3)$ & $111(3)$ \\
\hline $\mathrm{XCH}-3-1-37$ & $0.04896(0.00061)$ & $0.11911(0.00259)$ & $0.01765(0.00020)$ & $0.00576(0.00007)$ & $146(27)$ & $114(2)$ & $113(1)$ & $116(1)$ \\
\hline $\mathrm{XCH}-3-1-43$ & $0.04922(0.00212)$ & $0.11728(0.00635)$ & $0.01728(0.00022)$ & $0.00547(0.00005)$ & $158(96)$ & $113(6)$ & $110(1)$ & $110(1)$ \\
\hline XCH-3-1-47 & $0.05151(0.00123)$ & $0.12434(0.00436)$ & $0.01751(0.00023)$ & $0.00551(0.00009)$ & $264(56)$ & $119(4)$ & $112(1)$ & $111(2)$ \\
\hline XCH-3-1-09 & $0.04852(0.00282)$ & $0.12182(0.00865)$ & $0.01821(0.00029)$ & $0.00577(0.00006)$ & $124(139)$ & $117(8)$ & $116(2)$ & $116(1)$ \\
\hline $\mathrm{XCH}-3-1-24$ & $0.05117(0.00183)$ & $0.12768(0.00608)$ & $0.01810(0.00025)$ & $0.00563(0.00009)$ & $248(77)$ & $122(5)$ & $116(2)$ & $113(2)$ \\
\hline $\mathrm{XCH}-3-1-39$ & $0.04802(0.00215)$ & $0.12022(0.00675)$ & $0.01816(0.00025)$ & $0.00576(0.00006)$ & 100 (99) & $115(6)$ & $116(2)$ & $116(1)$ \\
\hline $\mathrm{XCH}-3-1-40$ & $0.05293(0.00371)$ & $0.13317(0.01143)$ & $0.01825(0.00033)$ & $0.00581(0.00011)$ & $326(170)$ & $127(10)$ & $117(2)$ & $117(2)$ \\
\hline $\mathrm{XCH}-3-1-41$ & $0.05043(0.00180)$ & $0.12476(0.00595)$ & $0.01794(0.00025)$ & $0.00590(0.00011)$ & $215(82)$ & $119(5)$ & $115(2)$ & $119(2)$ \\
\hline XCH-3-1-42 & $0.04931(0.00367)$ & $0.12730(0.01142)$ & $0.01872(0.00034)$ & $0.00592(0.00007)$ & $163(166)$ & $122(10)$ & $120(2)$ & $119(1)$ \\
\hline XCH-3-1-44 & $0.05231(0.00089)$ & $0.12966(0.00348)$ & $0.01798(0.00021)$ & $0.00567(0.00007)$ & 299 (39) & $124(3)$ & $115(1)$ & $114(1)$ \\
\hline XCH-3-1-48 & $0.05658(0.00347)$ & $0.14923(0.01121)$ & $0.01913(0.00030)$ & $0.00595(0.00006)$ & $475(150)$ & $141(10)$ & $122(2)$ & $120(1)$ \\
\hline \multicolumn{9}{|c|}{ Xiaojiang Type 2 MMEs } \\
\hline XC-4-1-02 & $0.04612(0.00200)$ & $0.10395(0.00534)$ & $0.01635(0.00018)$ & $0.00522(0.00005)$ & $4(96)$ & $100(5)$ & $105(1)$ & $105(1)$ \\
\hline XC-4-1-03 & $0.04609(0.00179)$ & $0.10124(0.00479)$ & $0.01593(0.00021)$ & $0.00512(0.00005)$ & $2(84)$ & $98(4)$ & $102(1)$ & $103(1)$ \\
\hline XC-4-1-04 & $0.04608(0.00123)$ & $0.10153(0.00354)$ & $0.01598(0.00019)$ & $0.00516(0.00006)$ & $2(53)$ & $98(3)$ & $102(1)$ & $104(1)$ \\
\hline XC-4-1-05 & $0.04918(0.00097)$ & $0.10940(0.00335)$ & $0.01613(0.00021)$ & $0.00503(0.00006)$ & $156(48)$ & $105(3)$ & $103(1)$ & $101(1)$ \\
\hline XC-4-1-06 & $0.04620(0.00248)$ & $0.10206(0.00644)$ & $0.01602(0.00021)$ & $0.00511(0.00005)$ & $8(117)$ & $99(6)$ & $102(1)$ & $103(1)$ \\
\hline XC-4-1-07 & $0.04609(0.00234)$ & $0.10344(0.00615)$ & $0.01628(0.00021)$ & $0.00520(0.00006)$ & $2(106)$ & $100(6)$ & $104(1)$ & $105(1)$ \\
\hline $\mathrm{XC}-4-1-08$ & $0.05550(0.00191)$ & $0.11659(0.00577)$ & $0.01524(0.00027)$ & $0.00487(0.00008)$ & $432(76)$ & $112(5)$ & $98(2)$ & $98(2)$ \\
\hline XC-4-1-09 & $0.04944(0.00270)$ & $0.11246(0.00750)$ & $0.01650(0.00023)$ & $0.00522(0.00004)$ & $169(116)$ & $108(7)$ & $105(1)$ & $105(1)$ \\
\hline
\end{tabular}


Table 1. (Continued)

\begin{tabular}{|c|c|c|c|c|c|c|c|c|}
\hline \multirow[b]{2}{*}{ Analysis } & \multicolumn{4}{|c|}{ Ratios (Common-Pb Corrected) } & \multicolumn{4}{|c|}{ Ages (Common-Pb Corrected, Ma) } \\
\hline & $\begin{array}{c}{ }^{207} \mathrm{~Pb} /{ }^{206} \mathrm{~Pb} \\
(1 \sigma)\end{array}$ & $\begin{array}{c}{ }^{207} \mathrm{~Pb} /{ }^{235} \mathrm{U} \\
(1 \sigma)\end{array}$ & $\begin{array}{c}{ }^{206} \mathrm{~Pb} /{ }^{238} \mathrm{U} \\
(1 \sigma)\end{array}$ & $\begin{array}{c}{ }^{208} \mathrm{~Pb} /{ }^{232} \mathrm{Th} \\
(1 \sigma)\end{array}$ & $\begin{array}{c}{ }^{207} \mathrm{~Pb} /{ }^{206} \mathrm{~Pb} \\
(1 \sigma)\end{array}$ & $\begin{array}{c}{ }^{207} \mathrm{~Pb} /{ }^{235} \mathrm{U} \\
(1 \sigma)\end{array}$ & $\begin{array}{c}{ }^{206} \mathrm{~Pb} /{ }^{238} \mathrm{U} \\
(1 \sigma)\end{array}$ & $\begin{array}{c}{ }^{208} \mathrm{~Pb} /{ }^{232} \mathrm{Th} \\
(1 \sigma)\end{array}$ \\
\hline XC-4-1-10 & $0.05091(0.00112)$ & $0.11302(0.00379)$ & $0.01610(0.00022)$ & $0.00503(0.00007)$ & $237(50)$ & $109(3)$ & $103(1)$ & $101(1)$ \\
\hline $\mathrm{XC}-4-1-13$ & $0.04608(0.00222)$ & $0.10518(0.00595)$ & $0.01655(0.00021)$ & $0.00530(0.00006)$ & $2(102)$ & $102(5)$ & $106(1)$ & $107(1)$ \\
\hline$X C-4-1-14$ & $0.05708(0.00425)$ & $0.12479(0.01113)$ & $0.01586(0.00026)$ & $0.00493(0.00004)$ & $495(153)$ & $119(10)$ & $101(2)$ & $99(1)$ \\
\hline $\mathrm{XC}-4-1-15$ & $0.05439(0.00373)$ & $0.12434(0.01023)$ & $0.01658(0.00026)$ & $0.00518(0.00005)$ & $387(150)$ & $119(9)$ & $106(2)$ & $104(1)$ \\
\hline XC-4-1-17 & $0.04744(0.00339)$ & $0.10418(0.00895)$ & $0.01593(0.00027)$ & $0.00506(0.00006)$ & $71(149)$ & $101(8)$ & $102(2)$ & $102(1)$ \\
\hline XC-4-1-18 & $0.04789(0.00334)$ & $0.10113(0.00857)$ & $0.01532(0.00027)$ & $0.00486(0.00005)$ & $94(159)$ & $98(8)$ & $98(2)$ & $98(1)$ \\
\hline XC-4-1-19 & $0.05362(0.00130)$ & $0.11744(0.00423)$ & $0.01589(0.00022)$ & $0.00540(0.00008)$ & $355(56)$ & $113(4)$ & $102(1)$ & $109(1)$ \\
\hline XC-4-1-20 & $0.05140(0.00055)$ & $0.11917(0.00229)$ & $0.01681(0.00018)$ & $0.00535(0.00006)$ & $259(22)$ & $114(2)$ & $107(1)$ & $108(1)$ \\
\hline XC-4-1-23 & $0.04675(0.00111)$ & $0.10534(0.00370)$ & $0.01634(0.00022)$ & $0.00520(0.00007)$ & $36(50)$ & $102(3)$ & $104(1)$ & $105(1)$ \\
\hline XC-4-1-24 & $0.05095(0.00337)$ & $0.11889(0.00926)$ & $0.01692(0.00024)$ & $0.00533(0.00004)$ & $239(136)$ & $114(8)$ & $108(2)$ & $107(1)$ \\
\hline XC-4-1-26 & $0.05069(0.00313)$ & $0.11230(0.00834)$ & $0.01607(0.00023)$ & $0.00506(0.00004)$ & $227(133)$ & $108(8)$ & $103(1)$ & $102(1)$ \\
\hline XC-4-1-27 & $0.05362(0.00095)$ & $0.11492(0.00321)$ & $0.01555(0.00019)$ & $0.00494(0.00006)$ & $355(37)$ & $110(3)$ & $99(1)$ & $100(1)$ \\
\hline XC-4-1-29 & $0.05168(0.00072)$ & $0.12019(0.00278)$ & $0.01687(0.00019)$ & $0.00540(0.00006)$ & $271(31)$ & $115(3)$ & $108(1)$ & $109(1)$ \\
\hline XC-4-1-31 & $0.04999(0.00075)$ & $0.10949(0.00267)$ & $0.01589(0.00018)$ & $0.00488(0.00005)$ & $195(34)$ & $105(2)$ & $102(1)$ & $98(1)$ \\
\hline XC-4-1-32 & $0.05319(0.00109)$ & $0.11889(0.00372)$ & $0.01621(0.00021)$ & $0.00514(0.00007)$ & $337(45)$ & $114(3)$ & $104(1)$ & $104(1)$ \\
\hline XC-4-1-33 & $0.05330(0.00080)$ & $0.11602(0.00288)$ & $0.01579(0.00019)$ & $0.00498(0.00006)$ & $342(34)$ & $111(3)$ & $101(1)$ & $100(1)$ \\
\hline XC-4-1-35 & $0.04613(0.00283)$ & $0.09787(0.00711)$ & $0.01539(0.00024)$ & $0.00491(0.00007)$ & $4(136)$ & $95(7)$ & $98(2)$ & $99(1)$ \\
\hline XC-4-1-36 & $0.04782(0.00248)$ & $0.10441(0.00656)$ & $0.01583(0.00021)$ & $0.00503(0.00005)$ & $91(118)$ & $101(6)$ & $101(1)$ & $101(1)$ \\
\hline $\mathrm{XC}-4-1-38$ & $0.05223(0.00118)$ & $0.11806(0.00400)$ & $0.01640(0.00022)$ & $0.00553(0.00008)$ & $295(49)$ & $113(4)$ & $105(1)$ & $111(2)$ \\
\hline XC-4-1-39 & $0.04685(0.00239)$ & $0.10630(0.00650)$ & $0.01646(0.00022)$ & $0.00524(0.00005)$ & 41 (117) & $103(6)$ & $105(1)$ & $106(1)$ \\
\hline $\mathrm{XC}-4-1-40$ & $0.04609(0.00132)$ & $0.10302(0.00369)$ & $0.01621(0.00018)$ & $0.00521(0.00005)$ & $2(62)$ & $100(3)$ & $104(1)$ & $105(1)$ \\
\hline XC-4-1-41 & $0.04608(0.00136)$ & $0.09907(0.00387)$ & $0.01559(0.00021)$ & $0.00503(0.00005)$ & $2(62)$ & $96(4)$ & $100(1)$ & $101(1)$ \\
\hline \multicolumn{9}{|c|}{ Longwangtang alkali feldspar granite } \\
\hline LHT-01-1-02 & $0.04783(0.00226)$ & $0.11745(0.00687)$ & $0.01781(0.00025)$ & $0.00565(0.00007)$ & $91(112)$ & $113(6)$ & $114(2)$ & $114(2)$ \\
\hline LHT-01-1-03 & $0.04820(0.00104)$ & $0.12041(0.00384)$ & $0.01812(0.00022)$ & $0.00570(0.00007)$ & $109(50)$ & $115(3)$ & $116(1)$ & $115(1)$ \\
\hline LHT-01-1-05 & $0.05049(0.00032)$ & $0.12615(0.00173)$ & $0.01812(0.00019)$ & $0.00575(0.00006)$ & $218(15)$ & $121(2)$ & $116(1)$ & $116(1)$ \\
\hline LHT-01-1-10 & $0.04852(0.00189)$ & $0.12032(0.00596)$ & $0.01799(0.00022)$ & $0.00570(0.00004)$ & $125(87)$ & $115(5)$ & $115(1)$ & $115(1)$ \\
\hline LHT-01-1-13 & $0.04693(0.00541)$ & $0.11292(0.01523)$ & $0.01745(0.00044)$ & $0.00555(0.00018)$ & $46(234)$ & $109(14)$ & $112(3)$ & $112(4)$ \\
\hline LHT-01-1-14 & $0.04806(0.00032)$ & $0.11512(0.00163)$ & $0.01737(0.00018)$ & $0.00557(0.00006)$ & $102(15)$ & $111(1)$ & $111(1)$ & $112(1)$ \\
\hline LHT-01-1-16 & $0.05012(0.00115)$ & $0.11971(0.00399)$ & $0.01732(0.00021)$ & $0.00603(0.00008)$ & $201(51)$ & $115(4)$ & $111(1)$ & $122(2)$ \\
\hline LHT-01-1-20 & $0.05027(0.00035)$ & $0.12446(0.00181)$ & $0.01796(0.00018)$ & $0.00574(0.00006)$ & 207 (17) & $119(2)$ & $115(1)$ & $116(1)$ \\
\hline LHT-01-1-22 & $0.05014(0.00029)$ & $0.12008(0.00151)$ & $0.01737(0.00017)$ & $0.00556(0.00006)$ & $201(13)$ & $115(1)$ & $111(1)$ & $112(1)$ \\
\hline LHT-01-1-23 & $0.04983(0.00130)$ & $0.12509(0.00460)$ & $0.01821(0.00023)$ & $0.00578(0.00009)$ & $187(60)$ & $120(4)$ & $116(1)$ & $116(2)$ \\
\hline LHT-01-1-24 & $0.04888(0.00248)$ & $0.12016(0.00739)$ & $0.01783(0.00023)$ & $0.00564(0.00005)$ & $142(116)$ & $115(7)$ & $114(1)$ & $114(1)$ \\
\hline LHT-01-1-26 & $0.04917(0.00032)$ & $0.12021(0.00168)$ & $0.01773(0.00018)$ & $0.00586(0.00006)$ & $156(17)$ & $115(2)$ & $113(1)$ & $118(1)$ \\
\hline LHT-01-1-27 & $0.05020(0.00028)$ & $0.12042(0.00150)$ & $0.01740(0.00017)$ & $0.00579(0.00006)$ & 204 (13) & $115(1)$ & $111(1)$ & $117(1)$ \\
\hline LHT-01-1-28 & $0.04841(0.00048)$ & $0.11791(0.00217)$ & $0.01767(0.00019)$ & $0.00575(0.00007)$ & $119(24)$ & $113(2)$ & $113(1)$ & $116(1)$ \\
\hline LHT-01-1-32 & $0.05023(0.00084)$ & $0.12370(0.00328)$ & $0.01786(0.00021)$ & $0.00596(0.00008)$ & $206(39)$ & $118(3)$ & $114(1)$ & $120(2)$ \\
\hline LHT-01-1-07 & $0.04631(0.00056)$ & $0.12020(0.00248)$ & $0.01883(0.00020)$ & $0.00603(0.00007)$ & $14(25)$ & $115(2)$ & $120(1)$ & $122(1)$ \\
\hline LHT-01-1-11 & $0.05011(0.00223)$ & $0.12803(0.00708)$ & $0.01853(0.00024)$ & $0.00585(0.00005)$ & $200(98)$ & $122(6)$ & $118(2)$ & $118(1)$ \\
\hline LHT-01-1-12 & $0.05265(0.00111)$ & $0.13693(0.00430)$ & $0.01887(0.00023)$ & $0.00637(0.00008)$ & $314(46)$ & $130(4)$ & $121(2)$ & $128(2)$ \\
\hline LHT-01-1-21 & $0.04610(0.00191)$ & $0.11807(0.00595)$ & $0.01858(0.00023)$ & $0.00594(0.00007)$ & $3(80)$ & $113(5)$ & $119(1)$ & $120(1)$ \\
\hline
\end{tabular}


Table 2. Major and trace element compositions of hosts and enclaves from Xiaojiang-Liangnong complexes and Longwangtang pluton in the Southeast Coast Magmatic Belt.

\begin{tabular}{|c|c|c|c|c|c|c|c|c|c|c|c|c|c|c|c|}
\hline \multirow{2}{*}{$\frac{\text { Type }}{\text { Sample }}$} & \multicolumn{7}{|c|}{ Xiaojiang hosts of Type 1 MMEs } & \multicolumn{8}{|c|}{ Xiaojiang Type 1 MMEs } \\
\hline & 20ZJ01 & 20ZJ02 & $20 Z J 23$ & $20 Z J 24$ & $20 \mathrm{ZJ} 25$ & $20 Z J 26$ & 20ZJ27 & 20ZJ03-1 & 20ZJ03-2 & 20ZJ04 & 20ZJ05 & 20ZJ06 & 20ZJ07 & 20ZJ29 & 20ZJ30 \\
\hline Rx type & \multicolumn{7}{|c|}{ Granodiorite } & \multicolumn{8}{|c|}{ Diorite } \\
\hline \multicolumn{16}{|l|}{$\mathrm{wt} \%$} \\
\hline $\mathrm{SiO}_{2}$ & 61.04 & 59.60 & 61.39 & 61.58 & 60.95 & 60.27 & 60.16 & 55.21 & 55.58 & 54.98 & 54.57 & 57.00 & 57.39 & 56.51 & 55.69 \\
\hline $\mathrm{TiO}_{2}$ & 0.85 & 0.92 & 0.94 & 0.91 & 0.93 & 0.96 & 0.96 & 1.12 & 1.12 & 1.10 & 1.31 & 1.05 & 1.02 & 1.10 & 1.00 \\
\hline $\mathrm{Al}_{2} \mathrm{O}_{3}$ & 16.65 & 17.04 & 16.57 & 16.38 & 16.33 & 16.54 & 16.44 & 17.70 & 17.93 & 17.76 & 16.93 & 17.24 & 16.98 & 17.27 & 16.84 \\
\hline $\mathrm{Fe}_{2} \mathrm{O}_{3}$ & 5.83 & 6.25 & 5.92 & 5.76 & 5.87 & 6.09 & 6.13 & 7.64 & 7.62 & 7.72 & 8.58 & 6.96 & 6.77 & 7.17 & 7.14 \\
\hline $\mathrm{MnO}$ & 0.10 & 0.10 & 0.09 & 0.09 & 0.09 & 0.09 & 0.09 & 0.11 & 0.11 & 0.15 & 0.12 & 0.11 & 0.14 & 0.10 & 0.11 \\
\hline $\mathrm{MgO}$ & 2.63 & 2.83 & 2.69 & 2.59 & 2.59 & 2.79 & 2.77 & 3.71 & 3.58 & 3.54 & 3.57 & 3.61 & 3.45 & 3.64 & 4.35 \\
\hline $\mathrm{CaO}$ & 5.00 & 5.52 & 4.63 & 4.46 & 4.62 & 4.91 & 4.89 & 6.68 & 6.71 & 6.00 & 6.65 & 6.26 & 5.88 & 6.21 & 6.80 \\
\hline $\mathrm{Na}_{2} \mathrm{O}$ & 3.40 & 3.39 & 3.09 & 3.07 & 3.15 & 3.24 & 3.32 & 3.80 & 3.82 & 4.10 & 3.71 & 3.67 & 3.57 & 3.75 & 3.55 \\
\hline $\mathrm{K}_{2} \mathrm{O}$ & 3.02 & 2.87 & 3.61 & 3.99 & 3.68 & 3.57 & 3.60 & 2.11 & 2.06 & 2.48 & 2.58 & 2.40 & 2.64 & 2.58 & 2.81 \\
\hline $\mathrm{P}_{2} \mathrm{O}_{5}$ & 0.30 & 0.31 & 0.29 & 0.28 & 0.29 & 0.31 & 0.31 & 0.41 & 0.40 & 0.36 & 0.47 & 0.36 & 0.35 & 0.39 & 0.32 \\
\hline L.O.I. & 0.96 & 0.99 & 0.58 & 0.56 & 0.95 & 0.52 & 0.48 & 0.60 & & & & 0.66 & 0.96 & 0.12 & 0.26 \\
\hline Total & 99.78 & 99.83 & 99.81 & 99.68 & 99.45 & 99.29 & 99.15 & 99.11 & 98.93 & 98.19 & 98.49 & 99.32 & 99.14 & 98.84 & 98.86 \\
\hline$* \mathrm{~A} / \mathrm{CNK}$ & 0.93 & 0.91 & 0.95 & 0.94 & 0.93 & 0.91 & 0.90 & 0.85 & 0.86 & 0.87 & 0.81 & 0.86 & 0.87 & 0.85 & 0.79 \\
\hline NK/A & 0.53 & 0.51 & 0.54 & 0.57 & 0.56 & 0.56 & 0.57 & 0.48 & 0.47 & 0.53 & 0.53 & 0.50 & 0.51 & 0.52 & 0.53 \\
\hline \multicolumn{16}{|l|}{ ppm } \\
\hline $\mathrm{La}$ & 43.3 & 40.0 & 41.9 & 50.7 & 55.6 & 50.4 & 43.7 & 37.9 & 41.8 & 43.8 & 43.1 & 37.7 & 39.7 & 46.4 & 43.1 \\
\hline $\mathrm{Ce}$ & 84.2 & 78.8 & 82.5 & 101 & 110 & 99.6 & 88.2 & 76.1 & 74.5 & 85.3 & 86.4 & 74.9 & 79.7 & 90.6 & 88.4 \\
\hline $\mathrm{Pr}$ & 9.20 & 8.34 & 8.84 & 11.0 & 11.8 & 10.8 & 9.43 & 8.38 & 9.24 & 9.47 & 9.82 & 8.41 & 8.55 & 9.96 & 9.68 \\
\hline $\mathrm{Nd}$ & 34.9 & 32.3 & 33.0 & 41.0 & 43.8 & 41.3 & 36.5 & 34.0 & 37.3 & 36.8 & 39.0 & 33.3 & 33.6 & 38.6 & 38.2 \\
\hline Sm & 5.96 & 5.63 & 5.53 & 6.92 & 7.40 & 6.93 & 6.33 & 6.16 & 7.10 & 6.38 & 6.76 & 5.82 & 5.92 & 6.48 & 6.93 \\
\hline $\mathrm{Eu}$ & 1.45 & 1.38 & 1.20 & 1.48 & 1.52 & 1.57 & 1.31 & 1.62 & 1.94 & 1.56 & 1.82 & 1.58 & 1.52 & 1.73 & 1.33 \\
\hline $\mathrm{Gd}$ & 4.83 & 4.43 & 4.61 & 5.56 & 5.93 & 5.67 & 4.92 & 4.87 & 6.39 & 5.25 & 5.39 & 4.68 & 4.60 & 5.12 & 5.36 \\
\hline $\mathrm{Tb}$ & 0.651 & 0.633 & 0.590 & 0.747 & 0.786 & 0.739 & 0.709 & 0.712 & 0.986 & 0.703 & 0.703 & 0.615 & 0.638 & 0.639 & 0.810 \\
\hline Dy & 3.32 & 3.23 & 2.99 & 3.77 & 4.01 & 3.80 & 3.58 & 3.65 & 5.46 & 3.54 & 3.50 & 3.04 & 3.17 & 3.11 & 4.21 \\
\hline Ho & 0.645 & 0.618 & & 0.711 & 0.777 & 0.730 & 0.683 & 0.703 & 1.06 & 0.680 & 0.672 & 0.574 & 0.585 & 0.559 & 0.798 \\
\hline $\mathrm{Er}$ & 1.76 & 1.69 & 1.62 & 1.98 & 2.13 & 2.03 & 1.87 & 1.89 & 2.95 & 1.86 & 1.82 & 1.52 & 1.57 & 1.50 & 2.15 \\
\hline $\mathrm{Tm}$ & 0.276 & 0.247 & 0.231 & 0.293 & 0.318 & 0.299 & 0.272 & 0.271 & 0.446 & 0.263 & 0.253 & 0.225 & 0.230 & 0.210 & 0.318 \\
\hline $\mathrm{Yb}$ & 1.70 & 1.62 & 1.53 & 1.85 & 2.01 & 1.90 & 1.84 & 1.67 & 2.75 & 1.69 & 1.55 & 1.41 & 1.45 & 1.30 & 2.09 \\
\hline $\mathrm{Lu}$ & 0.266 & 0.261 & 0.230 & 0.287 & 0.309 & 0.291 & 0.296 & 0.271 & 0.427 & 0.264 & 0.236 & 0.212 & 0.243 & 0.189 & 0.334 \\
\hline$(\mathrm{La} / \mathrm{Yb}) \mathrm{n}$ & 17.22 & 16.67 & 18.54 & 18.51 & 18.72 & 17.88 & 16.03 & 15.36 & 10.29 & 17.52 & 18.80 & 18.04 & 18.46 & 24.08 & 13.92 \\
\hline$(\mathrm{Yb}) \mathrm{n}$ & 6.85 & 6.54 & 6.17 & 7.47 & 8.09 & 7.68 & 7.42 & 6.72 & 11.08 & 6.81 & 6.24 & 5.69 & 5.86 & 5.25 & 8.44 \\
\hline $\mathrm{Sc}$ & 15.5 & 23.6 & 12.5 & 14.7 & 13.7 & 18.3 & 22.9 & 20.1 & 16.9 & 15.0 & 17.2 & 13.0 & 17.6 & 15.0 & 20.6 \\
\hline V & 95.3 & 147 & 63.5 & 95.1 & 109 & 104 & 84.2 & 190 & 167 & 143 & 184 & 141 & 173 & 132 & 138 \\
\hline $\mathrm{Cr}$ & 22.0 & 20.0 & 29.0 & 41.2 & 40.5 & 34.3 & 31.4 & 27.5 & 32.1 & 17.4 & 32.3 & 45.3 & 38.0 & 67.1 & 106 \\
\hline Co & 13.9 & 15.3 & 12.0 & 14.0 & 14.4 & 14.8 & 12.9 & 20.8 & 21.8 & 16.5 & 20.9 & 18.6 & 18.1 & 19.3 & 18.9 \\
\hline $\mathrm{Ni}$ & 12.3 & 11.7 & 13.1 & 16.5 & 16.7 & 15.5 & 16.5 & 17.4 & 19.6 & 13.3 & 20.1 & 21.0 & 20.7 & 26.8 & 27.1 \\
\hline $\mathrm{Ga}$ & 21.0 & 20.2 & 18.1 & 21.5 & 22.8 & 21.9 & 19.5 & 21.3 & 22.5 & 23.0 & 22.9 & 22.1 & 20.7 & 23.2 & 20.6 \\
\hline $\mathrm{Rb}$ & 93.6 & 74.6 & 126 & 156 & 154 & 143 & 122 & 62.1 & 71.7 & 99.0 & 73.5 & 76.0 & 76.0 & 89.0 & 108 \\
\hline $\mathrm{Sr}$ & 815 & 770 & 645 & 713 & 800 & 797 & 669 & 924 & 1042 & 858 & 1172 & 1010 & 827 & 1088 & 719 \\
\hline Y & 19.3 & 16.8 & 17.8 & 21.7 & 23.6 & 22.0 & 20.2 & 20.4 & 30.4 & 19.9 & 20.9 & 17.0 & 15.6 & 16.8 & 22.9 \\
\hline $\mathrm{Zr}$ & 244 & 208 & 337 & 346 & 414 & 412 & 296 & 192 & 221 & 164 & 214 & 212 & 204 & 242 & 168 \\
\hline $\mathrm{Nb}$ & 11.9 & 11.0 & 13.2 & 15.3 & 16.4 & 14.2 & 14.5 & 9.72 & 9.65 & 10.5 & 11.2 & 9.57 & 10.3 & 11.4 & 12.7 \\
\hline Cs & 2.42 & 2.00 & 2.59 & 3.36 & 4.80 & 3.86 & 3.69 & 2.15 & 2.58 & 3.78 & 1.97 & 2.33 & 2.05 & 1.93 & 3.08 \\
\hline $\mathrm{Ba}$ & 995 & 799 & 803 & 982 & 1032 & 976 & 895 & 707 & 879 & 622 & 1013 & 946 & 769 & 1031 & 618 \\
\hline $\mathrm{Hf}$ & 5.93 & 5.56 & 7.41 & 8.18 & 9.41 & 9.36 & 7.44 & 5.06 & 5.22 & 3.85 & 5.03 & 5.05 & 5.46 & 5.48 & 4.55 \\
\hline $\mathrm{Ta}$ & 0.597 & 0.795 & 0.671 & 0.845 & 0.915 & 0.748 & 1.08 & 0.601 & 0.411 & 0.400 & 0.452 & 0.438 & 0.705 & 0.516 & 0.963 \\
\hline $\mathrm{Pb}$ & 24.1 & 18.3 & 22.1 & 23.8 & 28.7 & 22.1 & 26.2 & 24.3 & 30.3 & 21.8 & 19.8 & 23.0 & 21.5 & 18.1 & 17.0 \\
\hline Th & 12.3 & 9.47 & 15.5 & 21.1 & 25.0 & 18.1 & 17.5 & 6.01 & 6.56 & 5.69 & 6.89 & 8.41 & 7.99 & 9.60 & 14.2 \\
\hline $\mathrm{U}$ & 2.97 & 2.10 & 3.92 & 5.33 & 6.16 & 4.55 & 4.71 & 2.52 & 2.04 & 1.57 & 2.35 & 2.01 & 1.73 & 2.21 & 3.57 \\
\hline $\mathrm{Sr} / \mathrm{Y}$ & 42.27 & 45.78 & 36.25 & 32.82 & 33.89 & 36.22 & 33.16 & 45.23 & 34.28 & 43.06 & 56.08 & 59.55 & 53.11 & 64.76 & 31.36 \\
\hline $10^{4} \mathrm{Ga} / \mathrm{Al}$ & 2.39 & 2.24 & 2.07 & 2.48 & 2.63 & 2.51 & 2.24 & 2.27 & 2.37 & 2.44 & 2.55 & 2.42 & 2.30 & 2.53 & 2.31 \\
\hline
\end{tabular}


Table 2. (Continued)

\begin{tabular}{|c|c|c|c|c|c|c|c|c|c|c|c|c|c|c|}
\hline \multirow{2}{*}{$\begin{array}{l}\text { Type } \\
\text { Sample }\end{array}$} & \multirow{2}{*}{$\begin{array}{c}\text { Type 1a } \\
20 Z J 31\end{array}$} & \multicolumn{6}{|c|}{ Xiaojiang hosts of Type 2 MMEs } & \multicolumn{7}{|c|}{ Xiaojiang Type 2 MMEs } \\
\hline & & 20ZJ12 & 20ZJ20 & 20ZJ33 & 20ZJ35 & $20 \mathrm{ZJ36}$ & 20ZJ37 & $20 Z J 13$ & 20ZJ14 & $20 Z J 16$ & 20ZJ19 & 20ZJ21 & 20ZJ22 & $20 \mathrm{ZJ34}$ \\
\hline Rx type & Gabbro & \multicolumn{6}{|c|}{ Alkali feldspar granite } & \multicolumn{7}{|c|}{ Monzogranite } \\
\hline \multicolumn{15}{|l|}{$\mathrm{wt} \%$} \\
\hline $\mathrm{SiO}_{2}$ & 45.43 & 75.76 & 75.06 & 73.74 & 77.91 & 77.09 & 78.53 & 64.91 & 64.81 & 64.42 & 62.64 & 61.98 & 63.73 & 67.87 \\
\hline $\mathrm{TiO}_{2}$ & 1.51 & 0.17 & 0.14 & 0.21 & 0.09 & 0.10 & 0.13 & 0.71 & 0.69 & 0.70 & 0.74 & 0.84 & 0.86 & 0.62 \\
\hline $\mathrm{Al}_{2} \mathrm{O}_{3}$ & 10.10 & 13.37 & 13.54 & 13.80 & 12.74 & 12.92 & 12.60 & 16.67 & 16.93 & 16.64 & 16.82 & 17.24 & 17.65 & 15.11 \\
\hline $\mathrm{Fe}_{2} \mathrm{O}_{3}$ & 19.24 & 0.68 & 0.51 & 1.32 & 0.35 & 0.33 & 0.49 & 3.79 & 3.78 & 4.27 & 4.76 & 4.94 & 4.89 & 4.23 \\
\hline $\mathrm{MnO}$ & 0.30 & 0.05 & 0.05 & 0.07 & 0.03 & 0.03 & 0.03 & 0.24 & 0.22 & 0.24 & 0.29 & 0.33 & 0.34 & 0.21 \\
\hline $\mathrm{MgO}$ & 13.80 & 0.00 & 0.00 & 0.17 & 0.00 & 0.00 & 0.00 & 1.18 & 1.20 & 1.10 & 1.42 & 1.46 & 1.49 & 1.23 \\
\hline $\mathrm{CaO}$ & 6.52 & 0.74 & 0.68 & 0.99 & 0.40 & 0.42 & 0.50 & 3.09 & 3.17 & 2.69 & 3.02 & 3.49 & 3.57 & 1.54 \\
\hline $\mathrm{Na}_{2} \mathrm{O}$ & 1.12 & 3.51 & 3.33 & 3.47 & 3.41 & 3.58 & 3.24 & 4.97 & 4.85 & 5.33 & 5.35 & 5.49 & 5.44 & 3.84 \\
\hline $\mathrm{K}_{2} \mathrm{O}$ & 0.72 & 4.74 & 5.21 & 4.54 & 4.65 & 4.74 & 4.21 & 2.64 & 2.57 & 2.68 & 2.41 & 2.03 & 1.87 & 3.81 \\
\hline $\mathrm{P}_{2} \mathrm{O}_{5}$ & 0.26 & 0.04 & 0.04 & 0.06 & 0.02 & 0.02 & 0.03 & 0.27 & 0.29 & 0.27 & 0.34 & 0.35 & 0.35 & 0.19 \\
\hline L.O.I. & & 0.32 & 0.42 & 0.28 & 0.28 & 0.22 & 0.16 & 0.50 & 0.64 & 0.42 & 0.66 & 0.48 & 0.60 & 0.42 \\
\hline Total & 98.99 & 99.38 & 98.98 & 98.65 & 99.88 & 99.45 & 99.92 & 98.96 & 99.15 & 98.75 & 98.44 & 98.61 & 100.76 & 99.06 \\
\hline $\mathrm{A} / \mathrm{CNK}$ & 0.70 & 1.09 & 1.09 & 1.11 & 1.12 & 1.10 & 1.16 & 1.00 & 1.02 & 1.00 & 1.00 & 0.98 & 1.01 & 1.14 \\
\hline NK/A & 0.26 & 0.82 & 0.82 & 0.77 & 0.84 & 0.85 & 0.79 & 0.66 & 0.64 & 0.70 & 0.68 & 0.65 & 0.62 & 0.69 \\
\hline \multicolumn{15}{|l|}{ ppm } \\
\hline $\mathrm{La}$ & 16.2 & 29.1 & 28.5 & 45.8 & 23.7 & 21.3 & 33.6 & 41.7 & 39.8 & 63.4 & 57.1 & 74.0 & 56.4 & 52.3 \\
\hline $\mathrm{Ce}$ & 34.0 & 56.4 & 51.6 & 82.4 & 43.3 & 42.7 & 63.3 & 87.7 & 79.8 & 151 & 112 & 146 & 125 & 106 \\
\hline $\operatorname{Pr}$ & 4.03 & 5.98 & 5.15 & 8.25 & 4.57 & 4.33 & 6.22 & 9.07 & 8.38 & 16.1 & 11.5 & 15.1 & 14.1 & 11.6 \\
\hline $\mathrm{Nd}$ & 16.6 & 21.1 & 17.7 & 28.5 & 15.8 & 15.1 & 21.3 & 33.5 & 32.3 & 55.2 & 40.7 & 57.3 & 56.2 & 43.9 \\
\hline $\mathrm{Sm}$ & 3.14 & 4.12 & 3.52 & 4.91 & 3.81 & 3.49 & 4.49 & 5.87 & 5.81 & 9.07 & 6.84 & 10.0 & 10.4 & 8.74 \\
\hline $\mathrm{Eu}$ & 0.748 & 0.404 & 0.484 & 0.727 & 0.206 & 0.156 & 0.276 & 1.29 & 1.38 & 1.30 & 1.19 & 2.01 & 2.11 & 0.646 \\
\hline $\mathrm{Gd}$ & 2.64 & 3.54 & 3.23 & 4.25 & 4.05 & 3.24 & 4.07 & 4.95 & 4.85 & 8.04 & 5.78 & 8.38 & 8.67 & 7.36 \\
\hline $\mathrm{Tb}$ & 0.377 & 0.681 & 0.556 & 0.636 & 0.802 & 0.661 & 0.780 & 0.803 & 0.802 & 1.19 & 0.944 & 1.30 & 1.39 & 1.24 \\
\hline Dy & 2.00 & 4.18 & 3.53 & 3.59 & 5.36 & 4.41 & 4.89 & 4.71 & 4.67 & 6.91 & 5.28 & 7.09 & 7.66 & 7.01 \\
\hline Но & 0.399 & 0.906 & 0.766 & 0.721 & 1.21 & 0.958 & 1.06 & 0.997 & 0.989 & 1.50 & 1.09 & 1.43 & 1.55 & 1.44 \\
\hline $\mathrm{Er}$ & 1.16 & 2.73 & 2.38 & 2.19 & 3.89 & 3.00 & 3.23 & 3.02 & 2.99 & 4.67 & 3.33 & 4.13 & 4.43 & 4.17 \\
\hline $\mathrm{Tm}$ & 0.184 & 0.480 & 0.404 & 0.346 & 0.697 & 0.535 & 0.559 & 0.512 & 0.502 & 0.808 & 0.566 & 0.651 & 0.696 & 0.668 \\
\hline $\mathrm{Yb}$ & 1.18 & 3.39 & 2.77 & 2.35 & 4.90 & 3.80 & 3.91 & 3.63 & 3.57 & 5.86 & 4.06 & 4.50 & 4.78 & 4.55 \\
\hline $\mathrm{Lu}$ & 0.189 & 0.531 & 0.420 & 0.366 & 0.761 & 0.637 & 0.658 & 0.644 & 0.610 & 1.03 & 0.695 & 0.792 & 0.837 & 0.776 \\
\hline$(\mathrm{La} / \mathrm{Yb}) \mathrm{n}$ & 9.23 & 5.81 & 6.95 & 13.18 & 3.27 & 3.79 & 5.81 & 7.76 & 7.53 & 7.31 & 9.49 & 11.11 & 7.97 & 7.77 \\
\hline$(\mathrm{Yb}) \mathrm{n}$ & 4.77 & 13.65 & 11.17 & 9.46 & 19.75 & 15.33 & 15.75 & 14.64 & 14.41 & 23.62 & 16.38 & 18.15 & 19.26 & 18.34 \\
\hline $\mathrm{Sc}$ & 20.4 & 16.3 & & 7.09 & 2.98 & 16.1 & & 13.9 & 7.62 & 6.69 & 6.89 & 13.9 & 20.7 & 6.68 \\
\hline V & 338 & & & & & & & & 14.3 & & & & & 10.6 \\
\hline $\mathrm{Cr}$ & 611 & & & & & & & & & & & & & \\
\hline Co & 73.0 & & 0.484 & 1.39 & & & & 2.75 & 2.83 & 3.20 & 2.99 & 2.99 & 3.14 & 3.68 \\
\hline $\mathrm{Ni}$ & 153 & & & & 2.34 & & & & & 0.801 & & & & \\
\hline $\mathrm{Ga}$ & 18.8 & 14.0 & 14.9 & 16.1 & 17.3 & 15.1 & 15.5 & 18.0 & 18.3 & 21.8 & 19.8 & 20.2 & 20.8 & 18.8 \\
\hline $\mathrm{Rb}$ & 41.0 & 244 & 292 & 187 & 331 & 274 & 280 & 249 & 236 & 297 & 259 & 227 & 207 & 176 \\
\hline $\mathrm{Sr}$ & 692 & 71.0 & 77.3 & 168 & 12.3 & 10.2 & 23.6 & 413 & 465 & 320 & 342 & 430 & 438 & 198 \\
\hline Y & 11.9 & 30.4 & 26.3 & 24.2 & 40.9 & 31.6 & 32.5 & 34.1 & 32.8 & 56.1 & 36.8 & 43.1 & 46.1 & 44.4 \\
\hline $\mathrm{Zr}$ & 36.0 & 109 & 87.4 & 146 & 104 & 87.9 & 109 & 236 & 218 & 282 & 192 & 221 & 233 & 306 \\
\hline $\mathrm{Nb}$ & 3.66 & 20.0 & 15.0 & 15.2 & 31.9 & 29.7 & 27.4 & 24.2 & 20.0 & 37.5 & 23.4 & 23.4 & 24.2 & 28.6 \\
\hline Cs & 1.59 & 2.25 & 2.37 & 1.52 & 1.92 & 1.76 & 2.20 & 8.73 & 7.36 & 8.03 & 9.20 & 9.81 & 8.04 & 2.31 \\
\hline $\mathrm{Ba}$ & 183 & 275 & 367 & 530 & 52.3 & 41.2 & 106 & 648 & 736 & 421 & 468 & 526 & 570 & 367 \\
\hline $\mathrm{Hf}$ & 1.16 & 3.67 & 2.75 & 4.28 & 4.74 & 4.10 & 4.80 & 5.87 & 5.49 & 6.69 & 4.83 & 5.64 & 5.91 & 8.66 \\
\hline $\mathrm{Ta}$ & 0.149 & 2.28 & 1.22 & 0.815 & 2.40 & 3.86 & 3.45 & 1.35 & 1.78 & 1.61 & 1.28 & 1.37 & 1.15 & 2.03 \\
\hline $\mathrm{Pb}$ & 4.84 & 16.2 & 22.3 & 20.0 & 19.3 & 17.3 & 17.4 & 11.3 & 11.3 & 15.0 & 11.6 & 14.5 & 13.7 & 12.3 \\
\hline Th & 1.58 & 27.2 & 23.4 & 18.2 & 35.7 & 38.0 & 29.8 & 19.7 & 17.4 & 20.3 & 12.1 & 10.2 & 11.0 & 24.5 \\
\hline $\mathrm{U}$ & 0.974 & 2.80 & 6.22 & 3.11 & 12.1 & 10.0 & 7.03 & 5.60 & 4.96 & 7.35 & 4.42 & 4.34 & 4.68 & 4.74 \\
\hline $\mathrm{Sr} / \mathrm{Y}$ & 58.21 & 2.34 & 2.94 & 6.92 & 0.30 & 0.32 & 0.73 & 12.11 & 14.17 & 5.71 & 9.30 & 9.99 & 9.52 & 4.46 \\
\hline $10^{4} \mathrm{Ga} / \mathrm{Al}$ & 3.52 & 1.98 & 2.08 & 2.20 & 2.57 & 2.20 & 2.32 & 2.04 & 2.04 & 2.47 & 2.22 & 2.21 & 2.23 & 2.35 \\
\hline
\end{tabular}


Table 2. (Continued)

\begin{tabular}{|c|c|c|c|c|c|c|c|c|c|c|c|c|}
\hline \multirow{2}{*}{$\begin{array}{l}\text { Type } \\
\text { Sample }\end{array}$} & \multicolumn{4}{|c|}{ Liangnong hosts of Type 1 MMEs } & \multicolumn{2}{|c|}{$\begin{array}{c}\text { Liangnong Type } 1 \\
\text { MMEs }\end{array}$} & \multicolumn{5}{|c|}{ Liangnong biotite granites } & \multirow{2}{*}{$\begin{array}{c}\text { Longwangtang } \\
\text { SLFP }\end{array}$} \\
\hline & 20ZJ50 & $20 Z J 52$ & $20 \mathrm{ZJ53}$ & 20ZJ56 & $20 Z J 54$ & $20 Z J 55$ & 20ZJ44 & $20 \mathrm{ZJ45}$ & 20ZJ46 & 20ZJ48 & 20ZJ47 & \\
\hline Rx type & \multicolumn{4}{|c|}{ Granodiorite } & \multicolumn{2}{|c|}{ Diorite } & \multicolumn{5}{|c|}{ Biotite granite } & $\begin{array}{c}\text { Alkali feldspar } \\
\text { granite }\end{array}$ \\
\hline \multicolumn{13}{|l|}{$\mathrm{wt} \%$} \\
\hline $\mathrm{SiO}_{2}$ & 62.87 & 61.43 & 62.72 & 63.03 & 54.62 & 54.65 & 70.05 & 71.68 & 72.68 & 73.92 & 77.02 & 72.87 \\
\hline $\mathrm{TiO}_{2}$ & 0.82 & 0.79 & 0.85 & 0.79 & 1.12 & 0.72 & 0.46 & 0.35 & 0.33 & 0.28 & 0.09 & 0.22 \\
\hline $\mathrm{Al}_{2} \mathrm{O}_{3}$ & 17.00 & 16.53 & 17.19 & 16.79 & 17.22 & 16.71 & 15.00 & 14.45 & 14.72 & 14.68 & 12.82 & 14.38 \\
\hline $\mathrm{Fe}_{2} \mathrm{O}_{3}$ & 4.83 & 4.86 & 5.05 & 4.96 & 7.17 & 7.61 & 2.55 & 1.93 & 1.87 & 1.58 & 0.34 & 1.17 \\
\hline $\mathrm{MnO}$ & 0.06 & 0.06 & 0.07 & 0.06 & 0.11 & 0.11 & 0.03 & 0.03 & 0.03 & 0.04 & 0.01 & 0.07 \\
\hline $\mathrm{MgO}$ & 2.56 & 2.47 & 2.66 & 2.55 & 4.40 & 4.63 & 0.98 & 0.63 & 0.64 & 0.43 & 0.00 & 0.29 \\
\hline $\mathrm{CaO}$ & 4.61 & 4.54 & 4.76 & 4.66 & 6.17 & 6.57 & 2.34 & 1.88 & 1.89 & 1.60 & 0.55 & 0.94 \\
\hline $\mathrm{Na}_{2} \mathrm{O}$ & 3.70 & 3.73 & 3.82 & 3.68 & 4.43 & 3.76 & 3.23 & 3.21 & 3.21 & 3.26 & 3.35 & 4.28 \\
\hline $\mathrm{K}_{2} \mathrm{O}$ & 3.34 & 3.35 & 3.29 & 3.30 & 2.51 & 3.40 & 4.77 & 4.69 & 4.56 & 4.86 & 4.74 & 4.82 \\
\hline $\mathrm{P}_{2} \mathrm{O}_{5}$ & 0.24 & 0.23 & 0.24 & 0.25 & 0.28 & 0.24 & 0.12 & 0.09 & 0.09 & 0.07 & 0.02 & 0.05 \\
\hline L.O.I. & 0.52 & 0.50 & 0.42 & 0.38 & & & 0.36 & 0.44 & 0.44 & 0.28 & 0.18 & \\
\hline Total & 100.55 & 98.49 & 101.06 & 100.44 & 98.01 & 98.39 & 99.87 & 99.37 & 100.47 & 101.00 & 99.12 & 99.09 \\
\hline $\mathrm{A} / \mathrm{CNK}$ & 0.94 & 0.92 & 0.93 & 0.93 & 0.81 & 0.77 & 1.02 & 1.05 & 1.08 & 1.08 & 1.10 & 1.03 \\
\hline NK/A & 0.57 & 0.59 & 0.57 & 0.57 & 0.58 & 0.59 & 0.70 & 0.72 & 0.70 & 0.73 & 0.83 & 0.85 \\
\hline \multicolumn{13}{|l|}{ ppm } \\
\hline $\mathrm{La}$ & 39.6 & 38.5 & 39.4 & 34.6 & 59.6 & 40.2 & 39.8 & 62.9 & 53.9 & 42.1 & 31.5 & 47.1 \\
\hline $\mathrm{Ce}$ & 79.5 & 76.1 & 78.1 & 69.4 & 116 & 78.8 & 85.2 & 105 & 92.3 & 82.1 & 51.2 & 88.2 \\
\hline $\operatorname{Pr}$ & 8.41 & 8.41 & 8.63 & 7.41 & 12.4 & 8.65 & 8.89 & 9.75 & 8.49 & 8.37 & 3.61 & 10.1 \\
\hline $\mathrm{Nd}$ & 32.6 & 32.5 & 33.4 & 29.0 & 46.8 & 33.5 & 32.9 & 32.5 & 29.1 & 29.0 & 8.74 & 34.3 \\
\hline Sm & 5.60 & 5.65 & 5.68 & 5.00 & 7.52 & 5.79 & 5.69 & 5.16 & 4.67 & 4.74 & 0.850 & 5.74 \\
\hline $\mathrm{Eu}$ & 1.30 & 1.40 & 1.43 & 1.27 & 1.45 & 1.27 & 0.938 & 0.878 & 0.743 & 0.793 & 0.086 & 1.01 \\
\hline $\mathrm{Gd}$ & 4.19 & 4.47 & 4.54 & 3.74 & 5.97 & 4.66 & 4.36 & 4.34 & 3.70 & 3.89 & 0.970 & 4.33 \\
\hline $\mathrm{Tb}$ & 0.566 & 0.564 & 0.562 & 0.497 & 0.74 & 0.580 & 0.635 & 0.563 & 0.523 & 0.518 & 0.098 & 0.700 \\
\hline Dy & 2.67 & 2.69 & 2.67 & 2.39 & 3.49 & 2.73 & 3.32 & 2.92 & 2.74 & 2.73 & 0.587 & 3.89 \\
\hline Но & 0.483 & 0.502 & 0.494 & 0.433 & 0.639 & 0.512 & 0.640 & 0.592 & 0.532 & 0.537 & 0.139 & 0.760 \\
\hline $\mathrm{Er}$ & 1.32 & 1.33 & 1.34 & 1.17 & 1.71 & 1.36 & 1.83 & 1.74 & 1.60 & 1.63 & 0.550 & 2.31 \\
\hline $\mathrm{Tm}$ & 0.185 & 0.190 & 0.192 & 0.161 & 0.236 & 0.199 & 0.299 & 0.287 & 0.264 & 0.274 & 0.123 & 0.350 \\
\hline $\mathrm{Yb}$ & 1.21 & 1.18 & 1.22 & 1.07 & 1.50 & 1.26 & 2.04 & 2.03 & 1.88 & 1.93 & 1.18 & 2.37 \\
\hline $\mathrm{Lu}$ & 0.196 & 0.186 & 0.182 & 0.166 & 0.225 & 0.192 & 0.328 & 0.326 & 0.324 & 0.309 & 0.234 & 0.370 \\
\hline$(\mathrm{La} / \mathrm{Yb}) \mathrm{n}$ & 22.03 & 22.04 & 21.82 & 21.81 & 26.82 & 21.55 & 13.22 & 20.95 & 19.32 & 14.76 & 18.07 & 13.43 \\
\hline$(\mathrm{Yb}) \mathrm{n}$ & 4.90 & 4.76 & 4.92 & 4.32 & 6.05 & 5.08 & 8.21 & 8.18 & 7.60 & 7.78 & 4.75 & 9.56 \\
\hline $\mathrm{Sc}$ & 17.7 & 12.5 & 18.6 & 11.2 & 18.0 & 20.1 & 16.2 & 16.2 & 15.0 & 6.76 & 17.0 & 42.9 \\
\hline V & 103 & 90.9 & 82.9 & 112 & 121 & 137 & 33.2 & & 20.9 & & & 8.74 \\
\hline $\mathrm{Cr}$ & 33.3 & 43.7 & 39.5 & 35.3 & 53.6 & 73.5 & 13.4 & 11.2 & 7.30 & 7.20 & & $<3$ \\
\hline Co & 12.8 & 14.0 & 14.1 & 12.9 & 23.5 & 18.0 & 4.90 & 4.56 & 3.65 & 3.27 & & 1.70 \\
\hline $\mathrm{Ni}$ & 23.1 & 24.6 & 25.1 & 23.5 & 42.4 & 35.8 & 8.23 & 7.55 & 5.48 & 5.28 & & 2.08 \\
\hline $\mathrm{Ga}$ & 21.9 & 23.8 & 23.9 & 21.4 & 26.2 & 23.5 & 18.4 & 20.5 & 18.4 & 20.3 & 18.8 & 16.5 \\
\hline $\mathrm{Rb}$ & 111 & 135 & 125 & 101 & 138 & 126 & 178 & 221 & 188 & 274 & 316 & 144 \\
\hline $\mathrm{Sr}$ & 796 & 950 & 980 & 800 & 919 & 874 & 439 & 398 & 338 & 335 & 28.8 & 180 \\
\hline $\mathrm{Y}$ & 13.6 & 15.3 & 15.2 & 12.2 & 19.8 & 15.9 & 19.8 & 19.8 & 17.1 & 18.6 & 6.25 & 22.1 \\
\hline $\mathrm{Zr}$ & 239 & 269 & 328 & 214 & 92.9 & 90.7 & 197 & 192 & 154 & 178 & 34.0 & 155 \\
\hline $\mathrm{Nb}$ & 8.60 & 8.58 & 8.10 & 7.33 & 11.2 & 6.63 & 15.2 & 14.0 & 13.6 & 15.1 & 20.6 & 15.7 \\
\hline Cs & 3.68 & 5.45 & 5.03 & 4.00 & 7.86 & 5.20 & 6.51 & 6.36 & 5.08 & 11.3 & 24.9 & 1.63 \\
\hline $\mathrm{Ba}$ & 792 & 996 & 992 & 823 & 594 & 823 & 769 & 640 & 534 & 596 & 23.4 & 1195 \\
\hline $\mathrm{Hf}$ & 6.74 & 6.58 & 7.57 & 5.85 & 2.82 & 2.74 & 6.1 & 5.82 & 5.37 & 5.77 & 2.41 & 5.37 \\
\hline $\mathrm{Ta}$ & 0.655 & 0.462 & 0.429 & 0.463 & 0.546 & 0.235 & 1.9 & 1.32 & 2.13 & 1.45 & 3.15 & 1.24 \\
\hline $\mathrm{Pb}$ & 16.1 & 14.9 & 13.9 & 12.3 & 19.6 & 13.2 & 16.3 & 20.9 & 18.3 & 22.9 & 25.0 & 38.9 \\
\hline Th & 13.9 & 19.5 & 16.0 & 13.2 & 14.3 & 12.0 & 32.5 & 49.6 & 40.0 & 58.2 & 24.8 & 19.1 \\
\hline $\mathrm{U}$ & 3.89 & 4.33 & 3.72 & 3.23 & 5.04 & 3.32 & 6.3 & 12.6 & 15.0 & 11.2 & 24.6 & 3.05 \\
\hline $\mathrm{Sr} / \mathrm{Y}$ & 58.47 & 61.96 & 64.36 & 65.40 & 46.44 & 55.07 & 22.15 & 20.08 & 19.76 & 17.98 & 4.61 & 8.16 \\
\hline $10^{4} \mathrm{Ga} / \mathrm{Al}$ & 2.43 & 2.72 & 2.63 & 2.41 & 2.88 & 2.65 & 2.31 & 2.67 & 2.36 & 2.61 & 2.77 & 2.16 \\
\hline
\end{tabular}


Table 3. Sr and Nd isotope compositions of hosts and enclaves from Xiaojiang-Liangnong complexes in the Southeast Coast Magmatic Belt.

\begin{tabular}{|c|c|c|c|c|c|c|c|c|c|c|c|c|}
\hline $\begin{array}{c}\text { Sample } \\
\text { No. }\end{array}$ & $\begin{array}{c}\mathbf{R b} \\
(\mathbf{p p m})\end{array}$ & $\begin{array}{c}\mathrm{Sr} \\
(\mathrm{ppm})\end{array}$ & $\begin{array}{c}\text { Sm } \\
(\mathbf{p p m})\end{array}$ & $\begin{array}{c}\text { Nd } \\
(\mathrm{ppm})\end{array}$ & ${ }^{87} \mathbf{R b} /{ }^{86} \mathrm{Sr}$ & ${ }^{87} \mathrm{Sr} /{ }^{86} \mathrm{Sr}$ & $2 \sigma$ & ${ }^{147} \mathrm{Sm} /{ }^{144} \mathrm{Nd}$ & ${ }^{143} \mathrm{Nd} /{ }^{144} \mathrm{Nd}$ & $2 \sigma$ & Isr & $\varepsilon N d(T)$ \\
\hline
\end{tabular}

Xiaojiang granodiorites

$\begin{array}{lllllllllllll}\text { 20ZJ02 } & 74.6 & 770 & 5.63 & 32.3 & 0.2805 & 0.708284 & 14 & 0.1058 & 0.512275 & 12 & 0.707845 & -5.8 \\ \text { 20ZJ27 } & 122 & 669 & 6.33 & 36.5 & 0.5280 & 0.708712 & 14 & 0.1049 & 0.512238 & 12 & 0.707887 & -6.5\end{array}$

Xiaojiang Type 1 MMEs

$\begin{array}{lllllllllllll}\text { 20ZJ07 } & 76.0 & 827 & 5.92 & 33.6 & 0.2661 & 0.708206 & 12 & 0.1065 & 0.512274 & 12 & 0.707790 & -5.9 \\ \text { 20ZJ30 } & 108 & 719 & 6.93 & 38.2 & 0.4349 & 0.708549 & 7 & 0.1097 & 0.512291 & 12 & 0.707869 & -5.6\end{array}$

Xiaojiang Type 1a MMEs

$\begin{array}{llllllllllllll}20 \mathrm{ZJ} 31 & 41 & 692 & 3.14 & 16.6 & 0.1716 & 0.708135 & 16 & 0.1144 & 0.512278 & 11 & 0.707867 & -5.9\end{array}$

Liangnong granodiorites

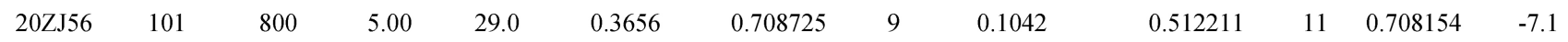

Liangnong Type 1 MMEs

$\begin{array}{lllllllllllll}20 Z J 54 & 138 & 919 & 7.52 & 46.8 & 0.4348 & 0.708777 & 2 & 0.0971 & 0.512143 & 12 & 0.708097 & -8.3\end{array}$

Xiaojiang alkali feldspar granites

\begin{tabular}{|c|c|c|c|c|c|c|c|c|c|c|c|}
\hline 20ZJ12 & 244 & 71 & 4.12 & 21.1 & 9.951 & 0.722894 & 9 & 0.1181 & 0.512314 & 12 & 0.707338 \\
\hline 20ZJ36 & 274 & 10.2 & 3.49 & 15.1 & 77.78 & N.A. & & 0.1397 & 0.512290 & 12 & N.A. \\
\hline
\end{tabular}

Xiaojiang Type 2 MMEs

$\begin{array}{lllllllllllll}20 \mathrm{ZJ} 14 & 236 & 465 & 5.81 & 32.3 & 1.470 & 0.709748 & 7 & 0.1088 & 0.512319 & 12 & 0.707451 & -5.0 \\ 20 \mathrm{ZJ} 21 & 227 & 430 & 10.0 & 57.3 & 1.529 & 0.709725 & 8 & 0.1055 & 0.512307 & 12 & 0.707335 & -5.2\end{array}$

Liangnong biotite granites

\begin{tabular}{|c|c|c|c|c|c|c|c|c|c|c|c|}
\hline 20ZJ44 & 178 & 439 & 5.69 & 32.9 & 1.174 & 0.709719 & 0.1046 & 0.512275 & 13 & 0.707884 & -5.8 \\
\hline 20ZJ47 & 316 & 28.8 & 0.85 & 8.74 & 31.77 & N.A. & 0.0588 & 0.512189 & 12 & N.A. & -7.0 \\
\hline
\end{tabular}

(a) Amphibole

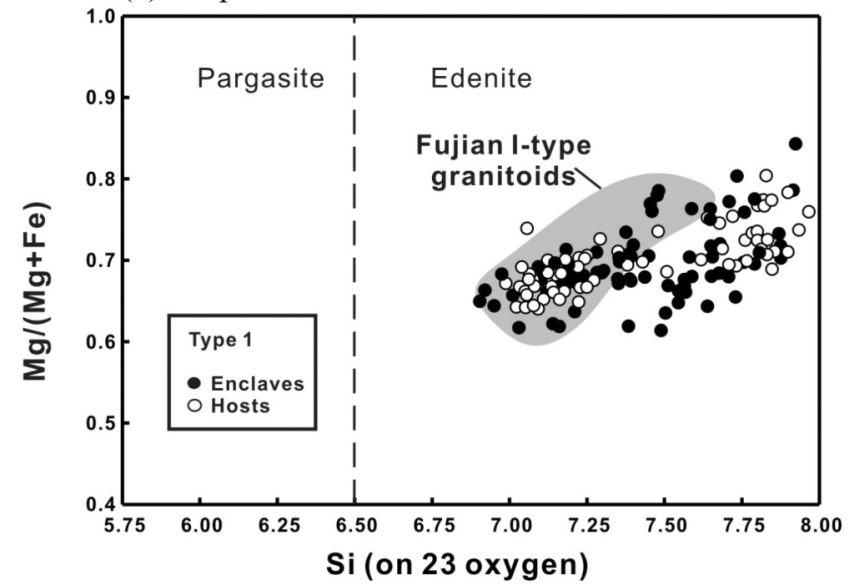

(b) Biotite

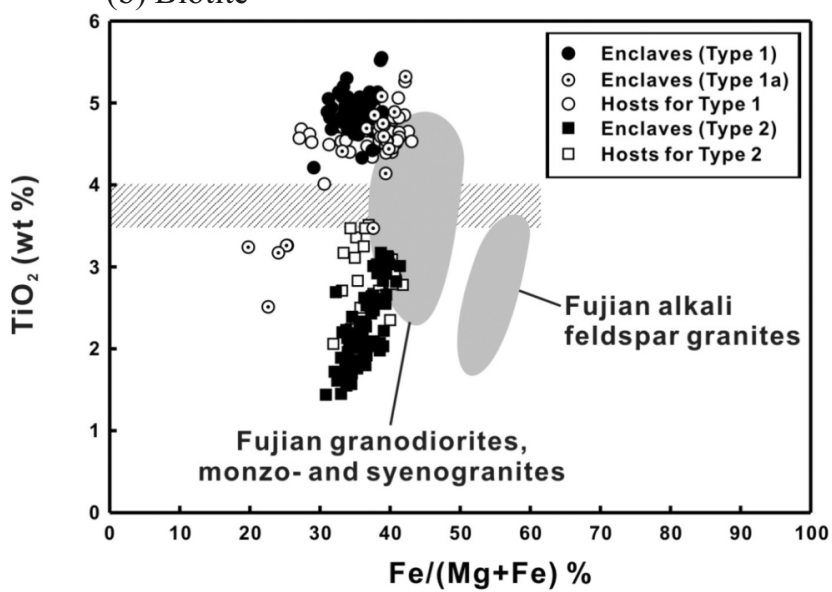

Fig. 3. Compositions of (a) amphibole and (b) biotite for the Xiaojiang-Liangnong igneous complexes as compared with those of the Fujian I-type granitoids. The stippled bar in biotite distribution marks the compositional gap between Type $1 \mathrm{MMEs}$ host $\left(\mathrm{TiO}_{2}>4.0 \mathrm{wt} \%\right)$ and Type $2 \mathrm{MMEs}$-host $\left(\mathrm{TiO}_{2}<3.5 \mathrm{wt} \%\right)$ pairs. Data sources of amphibole and biotite are taken from Yen (2005) and distribution fields of Fujian granitoids are from Lai (1995). 
$<0.11 \mathrm{wt} \% \mathrm{U}_{3} \mathrm{O}_{8}$ for Xiaojiang granodiorite and $67.1-$ $62.1 \mathrm{wt} \% \mathrm{ZrO}_{2}, 1.75-0.98 \mathrm{wt} \% \mathrm{HfO}_{2},<0.10 \mathrm{wt} \% \mathrm{U}_{3} \mathrm{O}_{8}$, and $<0.05 \mathrm{wt} \% \mathrm{ThO}_{2}$ for Longwangtang alkali feldspar granite (Table 4).

\section{AGE RESULTS}

${ }^{40} \mathrm{Ar} /{ }^{39} \mathrm{Ar}$ biotite ages reported previously are $101 \mathrm{Ma}$ for granodiorite and monzogranite from Liangnong, and $110 \mathrm{Ma}$ for biotite granite and alkali-feldspar granite from Longwangtang (Chen et al. 1991). More recently, Chen et al.
(2005) reported that the upper limit for Liangnong granites is ca. 113 - 110 Ma. Here we show the ${ }^{206} \mathrm{~Pb} /{ }^{238} \mathrm{U}$ vs. ${ }^{207} \mathrm{~Pb} /{ }^{235} \mathrm{U}$ concordia diagrams, accompanied with representative zircon CL images (Figs. 4a-c), for a granodiorite (XCH-3-1, or host of Type 1 MMEs) and a Type 2 MMEs (XC-4-1) from Xiaojiang, and an alkali feldspar granite from Longwangtang (LHT-01-1, or host of Type 2 MMEs). These ages shall be particularly important to unravel the formation time of granitoids in N Zhejiang and provide information for knowing the difference between the ${ }^{40} \mathrm{Ar} /{ }^{39} \mathrm{Ar}$ biotite and $\mathrm{U}-\mathrm{Pb}$ zircon age systems.

Table 4. Electron microprobe analyses in representative zircons from Xiaojiang and Longwangtang complexes of S China.

\begin{tabular}{|c|c|c|c|c|c|}
\hline Sample No. & $\begin{array}{c}{ }^{206} \mathrm{~Pb} /{ }^{238} \mathrm{U} \text { age } \\
\text { (Ma) }\end{array}$ & $\begin{array}{c}\mathrm{ZrO}_{2} \\
(\mathrm{wt} \%)\end{array}$ & $\begin{array}{c}\mathrm{HfO}_{2} \\
(\mathrm{wt} \%)\end{array}$ & $\begin{array}{c}\mathrm{U}_{3} \mathrm{O}_{8} \\
\left(w \mathrm{wt}^{2}\right)\end{array}$ & $\begin{array}{l}\mathrm{ThO}_{2} \\
(\mathrm{wt} \%)\end{array}$ \\
\hline \multicolumn{6}{|c|}{ Xiaojiang granodiorite } \\
\hline XCH-3-1-08 & 110 & 66.77 & 1.22 & 0.01 & b.d.l. \\
\hline XCH-3-1-18 & 110 & 66.83 & 1.80 & 0.11 & b.d.l. \\
\hline $\mathrm{XCH}-3-1-18 \mathrm{R}$ & & 66.47 & 1.81 & 0.06 & b.d.l. \\
\hline $\mathrm{XCH}-3-1-23$ & 108 & 66.18 & 1.52 & 0.05 & b.d.1. \\
\hline $\mathrm{XCH}-3-1-23 \mathrm{R}$ & & 65.66 & 1.40 & 0.02 & b.d.l. \\
\hline $\mathrm{XCH}-3-1-26$ & 110 & 63.69 & 1.27 & 0.04 & b.d.l. \\
\hline XCH-3-1-47 & 112 & 62.81 & 1.38 & 0.04 & b.d.l. \\
\hline \multicolumn{6}{|c|}{ Xiaojiang Type 2 MMEs } \\
\hline XC-4-1-04 & 102 & 65.63 & 1.83 & 0.03 & 0.05 \\
\hline XC-4-1-19 & 107 & 63.83 & 1.41 & 0.02 & b.d.1. \\
\hline XC-4-1-19R & & 64.34 & 1.38 & 0.05 & b.d.1. \\
\hline XC-4-1-17 & 98 & 66.52 & 1.37 & 0.06 & 0.06 \\
\hline XC-4-1-31 & 104 & 66.26 & 1.30 & 0.13 & 0.17 \\
\hline XC-4-1-39 & 105 & 63.86 & 1.26 & 0.13 & 0.16 \\
\hline XC-4-1-39R & & 64.36 & 1.31 & 0.08 & 0.15 \\
\hline \multicolumn{6}{|c|}{ Longwangtang alkali feldspar granite } \\
\hline LHT-1-1-02 & 114 & 64.82 & 1.10 & 0.03 & 0.03 \\
\hline LHT-1-1-02R & & 62.13 & 1.47 & 0.05 & b.d.l. \\
\hline LHT-1-1-10 & 115 & 63.74 & 1.32 & 0.04 & b.d.l. \\
\hline LHT-1-1-13 & 112 & 67.01 & 1.75 & 0.10 & 0.05 \\
\hline LHT-1-1-22 & 111 & 67.12 & 1.38 & 0.02 & b.d.l. \\
\hline LHT-1-1-22C & & 66.58 & 0.98 & b.d.l. & b.d.l. \\
\hline LHT-1-1-28 & 113 & 63.65 & 1.38 & 0.05 & b.d.l. \\
\hline LHT-1-1-29 & & 66.68 & 1.50 & 0.08 & b.d.l. \\
\hline
\end{tabular}

Note: $R=$ rim; $C=$ core.

b.d.l.: below detection limit. 
(a) $\mathrm{XCH}-3-1$

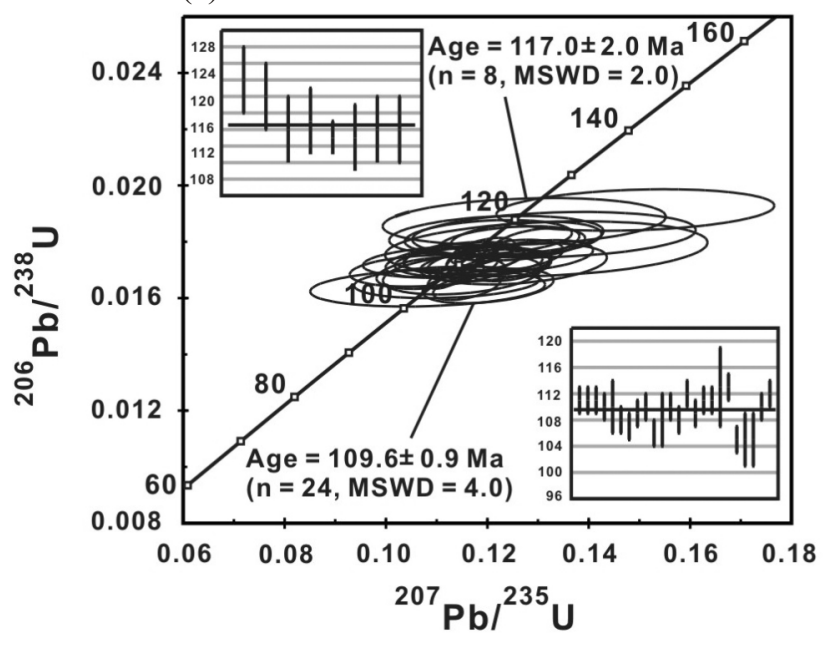

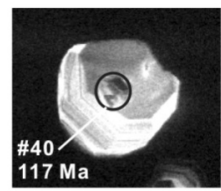
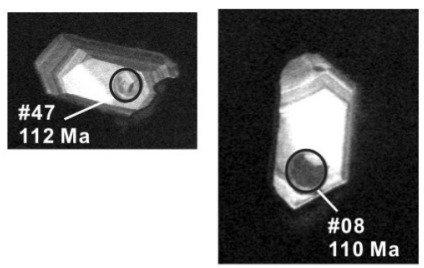

(b) XC-4-1
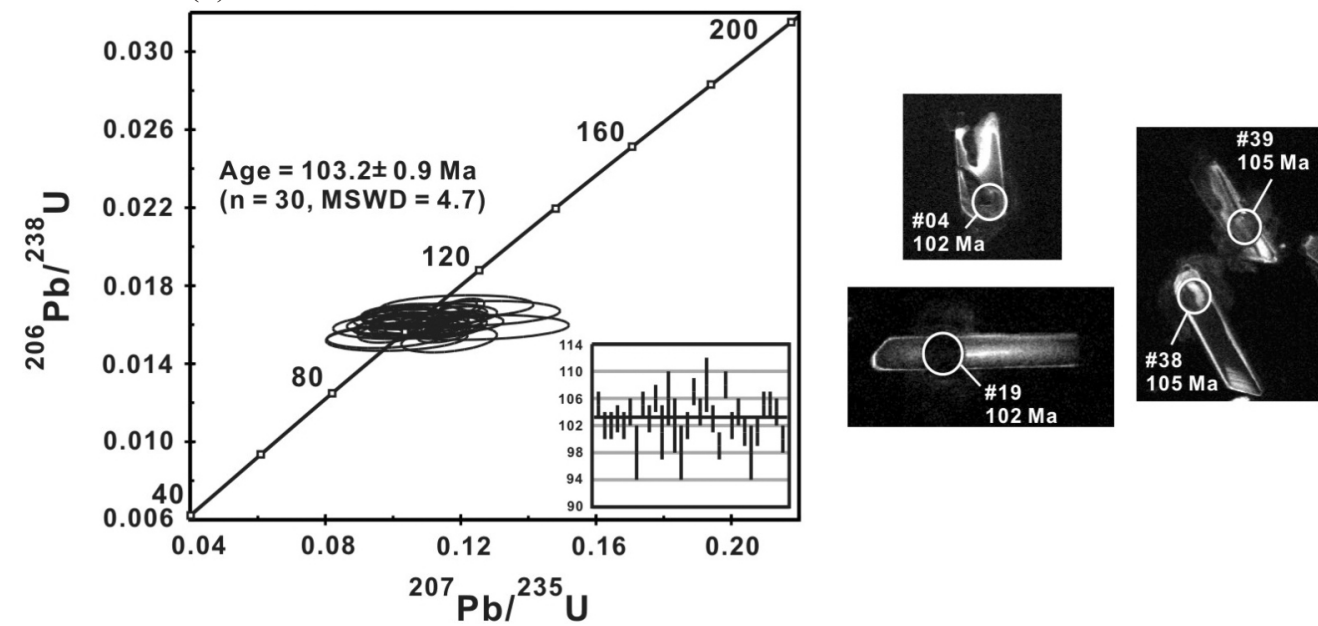

(c) LHT-01-1
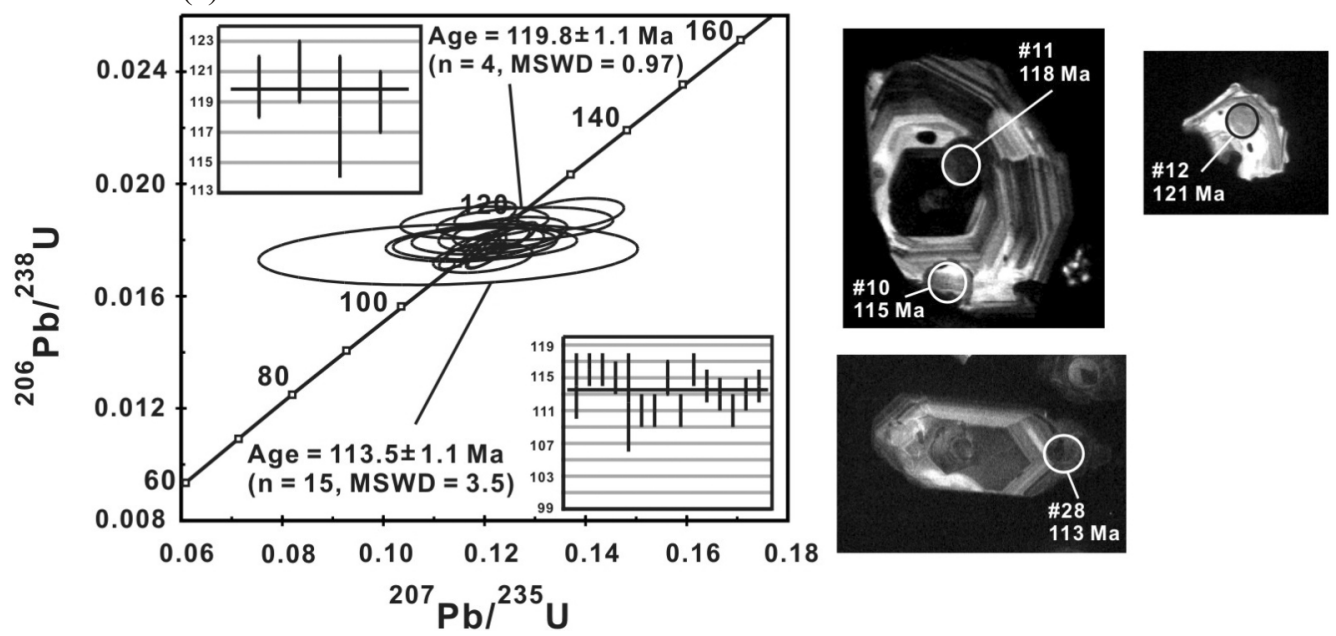

Fig. 4. Concordia of U-Pb zircon ages and cathodoluminescence (CL) images of dated zircons for (a) Xiaojiang granodiorite; (b) Xiaojiang Type 2 MMEs, and (c) Longwangtang alkali feldspar granite. The spot size of laser beam bombardment on zircon CL images is $\sim 40 \mu m$. Note the smaller zircons in the Type 2 MMEs yield only one single age and the greater zircons in both the granodiorite and alkali feldspar granite hosts yield two age groups, with the older one as the inherited age. 
Zircons from sample XCH-3-1 are mostly euhedral with good prism and pyramid faces, up to $150-300 \mu \mathrm{m}$ long, and have an elongation ratio (length to width ratio) of $2: 1$ to $3: 1$. Most grains are colorless and have the transparency varying from clear to cloudy. Euhedral concentric zoning is common in most crystals and a few inherited dark cores are observed in the CL images. On the basis of thirty-two grain analyses, sample $\mathrm{XCH}-3-1$ yields two significant $\mathrm{U}-\mathrm{Pb}$ zircon ages: $117.0 \pm$ $2.0(\mathrm{n}=8 ; \mathrm{MSWD}=2.0)$ and $109.6 \pm 0.9 \mathrm{Ma}(\mathrm{n}=24 ; \mathrm{MSWD}$ $=4.0$ ) (Fig. 4a). The old age, measured either from dark cores of the crystal in CL images or rounded single grain, is interpreted as the inherited zircon age. On the other hand, zircons from Xiaojiang Type 2 MMEs (sample XC-4-1) are significantly long-prismatic and euhedral with common concentric zoning. They are colorless, relatively transparent and mainly between $110-200 \mu \mathrm{m}$ in length with an elongation ratio of $3: 1$ to $4: 1$. Thirty zircon separates yield a single $\mathrm{U}-\mathrm{Pb}$ age of $103.2 \pm 0.9 \mathrm{Ma}(\mathrm{n}=30$; MSWD = 4.7) (Fig. 4b). With the large amount of zircon analyzed, sample XC-4-1 is demonstrated to be free from inheritance of zircons.

Zircon separates from Longwangtang alkali feldspar granite (sample LHT-01-1) are mostly euhedral and range between 150 and $300 \mu \mathrm{m}$ in length with an elongation ratio of $2: 1$ to $3: 1$. They are mainly colorless with the transparency varying from clear to cloudy. Although some grains show significant oscillatory zones, the majority of Longwangtang zircon samples are virtually non-luminescent. A total number of nineteen zircon grains have been subjected to the $\mathrm{U}-\mathrm{Pb}$ zircon age dating. Among them, fifteen analyses can define a $\mathrm{U}-\mathrm{Pb}$ age of $113.5 \pm 1.1 \mathrm{Ma}(\mathrm{n}=15$; MSWD $=$ 3.5 ), and four analyses yield an older age of $119.8 \pm 1.1 \mathrm{Ma}$ $(\mathrm{n}=4$; MSWD = 0.97) (Fig. 4c). Again, the latter is interpreted to be inherited zircon age.

\section{GEOCHEMISTRY}

Types 1 and 2 MMEs have a narrow range of $\mathrm{SiO}_{2}$ content varying from $55-57$ and $62-68 \mathrm{wt} \%$, respectively; in addition, the Type 1a (gabbroic) MMEs has an extremely low $\mathrm{SiO}_{2}$ content (45.4 wt\%) (Table 2). With the $\mathrm{SiO}_{2}$ content varying from $60-63 \mathrm{wt} \%$ for granodiortie and $70-$ $79 \mathrm{wt} \%$ for biotite granite-alkali feldspar granite, their host rocks are apparently marked with paucity of $\mathrm{SiO}_{2}$ at 63 $70 \mathrm{wt} \%$. The enclave $\left(\mathrm{SiO}_{2}=54-62 \mathrm{wt} \%\right)$-monzogranite host $\left(\mathrm{SiO}_{2}=70-73 \mathrm{wt} \%\right)$ pair in Beizhang is closer to the Type 2 MMEs-host in the geochemical aspect (Chen et al. 2005), conforming to the mineralogical classification scheme mentioned above. In the $\mathrm{K}_{2} \mathrm{O}$ vs. $\mathrm{SiO}_{2}$ plots (Fig. 5), all the studied rocks belong to high-K calc-alkaline series, except the Type 1a and Type 2 MMEs, which are mostly in the medium-K field. Granitoids in N Zhejiang are metaluminous to slightly peraluminous with molecular $\mathrm{A} / \mathrm{CNK}$ ratio ranges from 0.90 to 0.95 in the Type 1 hosts and from 1.02 to 1.16 in Type 2 hosts. The enclaves have A/CNK values lower than that of the respective host granites, i.e., 0.77 to 0.87 in Type 1 MMEs and 0.98 to 1.14 in Type 2 MMEs (Table 2). In the Harker's variation diagrams (Fig. 6), all the MMEs and host granites (except Type 1a gabbroic MMEs) can define a decreasing trend for $\mathrm{Al}_{2} \mathrm{O}_{3}, \mathrm{TiO}_{2}, \mathrm{CaO}, \mathrm{MgO}, \mathrm{P}_{2} \mathrm{O}_{5}$, and $\mathrm{Sr}$, but the increasing trend for $\mathrm{Rb}$ and $\mathrm{Nb}$ is not clear. Particularly in the $\mathrm{Zr}, \mathrm{Ba}$, and $\mathrm{La}$ vs. $\mathrm{SiO}_{2}$ plots (Fig. 7), decreasing variation trends are obvious only in the biotite granite-alkali feldspar granite association. Moreover, the primative mantlenormalized multi-element variation diagrams show distinctive distribution patterns for granodiorites (mafic granites) and biotite granites-alkali feldspar granites (felsic granites), with the latter being relatively enriched in $\mathrm{Rb}, \mathrm{Th}, \mathrm{U}, \mathrm{Nb}, \mathrm{Ta}$, HREE, and depleted in $\mathrm{Ba}, \mathrm{Sr}, \mathrm{P}, \mathrm{Eu}$, and Ti (Fig. 8). It is noted that both the Types 1 and 2 MMEs have similar distribution patterns overlapping the granodiorite field if they were depicted in the same diagram.

Age-corrected $\mathrm{Sr}$ and $\mathrm{Nd}$ isotopic ratio plots for MMEs and host rocks of Xiaojiang-Liangnong complexes are shown in Fig. 9 ( $n=13$ in Table 3), in which those for Cretaceous granitoids in SCMB are also presented (Lapierre et al. 1997; Chen et al. 2004). When all these data are compared, two important features are noted. (1) The enclaves generally exhibit similar $\mathrm{Sr}$ and $\mathrm{Nd}$ isotopic compositions to their host rocks: Type 1 MMEs (including Type 1a gabbroic enclave) in Xiaojiang $(\mathrm{Isr}=0.7078 \sim 0.7079 ; \varepsilon \mathrm{Nd}(\mathrm{T})=-5.6 \sim-5.9)$ and their hosts $(\mathrm{Isr}=0.7078 \sim 0.7079 ; \varepsilon \mathrm{Nd}(\mathrm{T})=-5.8 \sim-6.5)$; Type 1 MMEs in Liangnong $(\mathrm{Isr}=0.7081 ; \varepsilon \mathrm{Nd}(\mathrm{T})=-8.3$ ) and their hosts $(\mathrm{Isr}=0.7082 ; \varepsilon \mathrm{Nd}(\mathrm{T})=-7.1)$; Type $2 \mathrm{MMEs}$ in Xiaojiang ( $\mathrm{Isr}=0.7073 \sim 0.7075 ; \varepsilon \mathrm{Nd}(\mathrm{T})=-5.0 \sim-5.2$ ) and their hosts (Isr $=0.7073 ; \varepsilon \mathrm{Nd}(\mathrm{T})=-5.2 \sim-6.0)$. (2) In general, all the studied samples are comparable with the I-type granitoids in the SCMB. Differences of Sr-Nd isotopic compositions between Liangnong complex ( $\mathrm{Isr}=0.7079$ $\sim 0.7082 ; \varepsilon \mathrm{Nd}(\mathrm{T})=-5.8 \sim-7.1$ ) and Xiaojiang complex (Isr $=0.7073 \sim 0.7079 ; \varepsilon \mathrm{Nd}(\mathrm{T})=-5.2 \sim-6.5)$ are small, they as a whole are more enriched than the Fujian granitoids.

\section{DISCUSSION}

\subsection{Zircon Saturation Temperature of Granites and Their Cooling Rates}

In the granitic suites, $\mathrm{Zr}$ (usually accompanied by $\mathrm{Ba}$ and REE) rises from low values to a maximum at a little below $70 \% \mathrm{SiO}_{2}$, then falls back to low values for high temperature suites. Only in the low temperature I-type or the S-type granite suites, $\mathrm{Zr}$ has a linear variation (usually negatively correlated) against $\mathrm{SiO}_{2}$ (Chappell et al. 1998). Based on the restite model, Chappell (2003a) advocated that the inflected trends of $\mathrm{Zr}$ is evidence showing that high-temperature I-type granite magma was not saturated in zircon during the first part of its evolution, but became saturated near the point of $\mathrm{Zr}$ inflection. In this regard, the high temperature granite magmas were produced by the partial melting of mafic 
source rocks, and the low temperature granites resulted from the partial melting of quartzofeldspathic rocks. Therefore, the use of zircon saturation temperature based on age inheritance, along with the bulk rock geochemical distinctions, provides an effective test of whether a particular I-type suite evolved either by fractionation of restite or in some other way (Chappell 2003b).

In N Zhejiang, verifying the existence of inherited cores on zircon for both the granodiorite and the alkali feldspar granite is difficult unless a smaller beam size of age determination (such as using the ion probe) is applied. Judging from the slight age difference in the few grains that have been determined with the core-rim sites (Figs. 4a, c), we suggest that older ages obtained from these two host rocks represent the core age of some zircon grains. A similar phenomenon is presented in the neighboring Julipin rhyolites, in which the $101 \mathrm{Ma}$ zircons are often accompanied by a $130 \mathrm{Ma}$ core (Chen et al. 2008). On the other hand, the elongated zir-

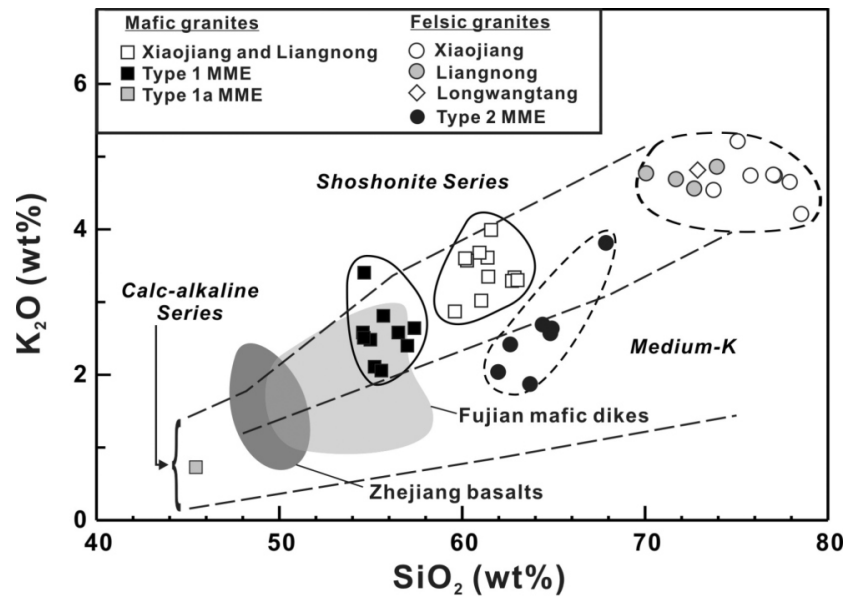

Fig. 5. The $\mathrm{K}_{2} \mathrm{O}$ vs. $\mathrm{SiO}_{2}$ diagram for the Xiaojiang-Liangnong granitoids (mafic granites: $\mathrm{SiO}_{2}<70 \mathrm{wt} \%$; felsic rocks: $\mathrm{SiO}_{2}>70 \mathrm{wt} \%$ ) and adjacent pluton, and their enclaves. Distribution fields of Fujian mafic dikes and Zhejiang basalts are taken from Chen et al. (2008). (a)

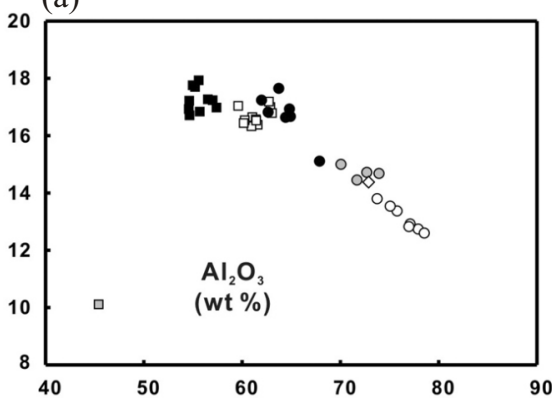

(d)

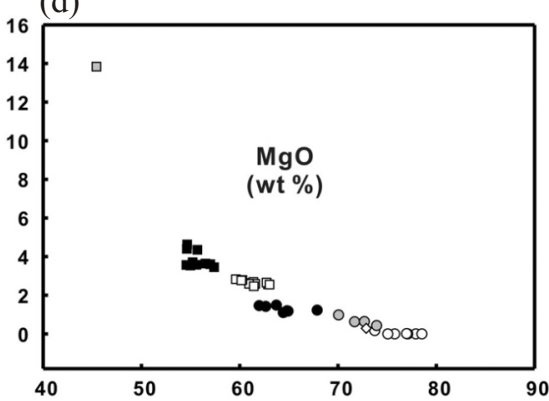

(g)

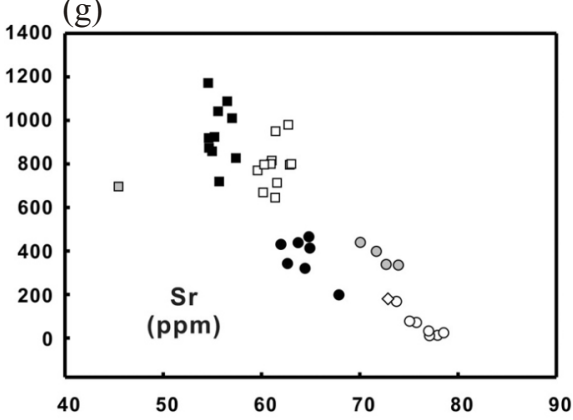

(b)

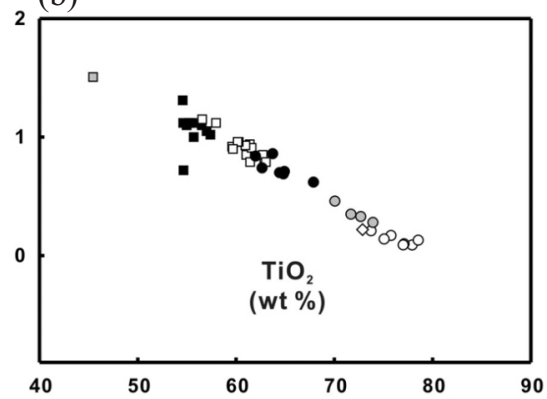

(e)
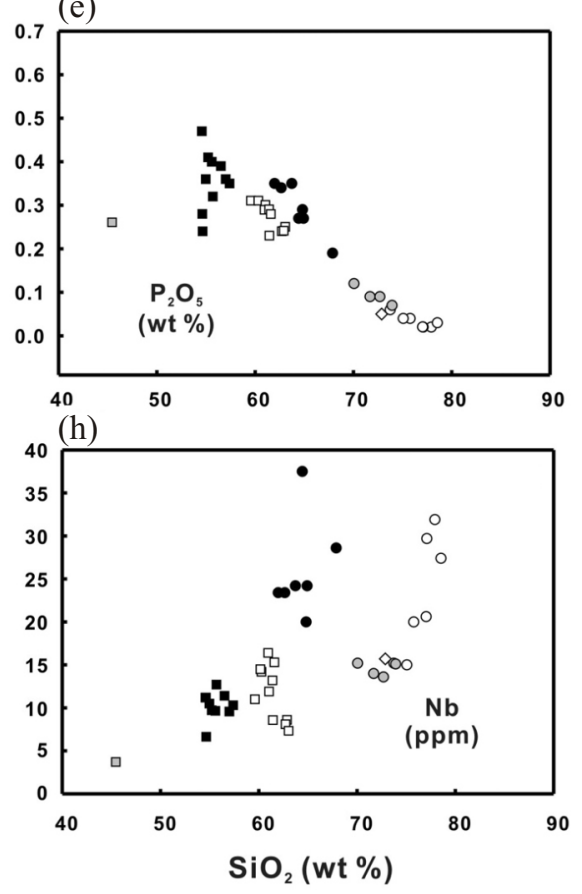

(c)
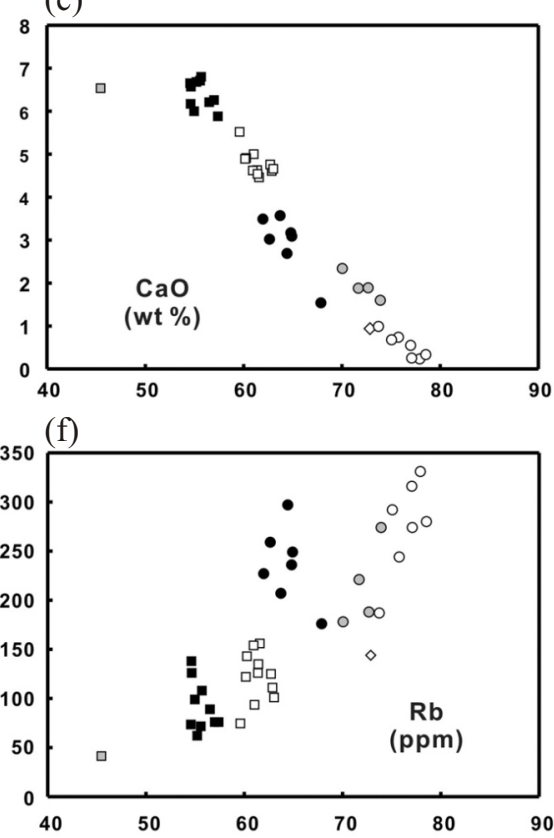

Fig. 6. Harker's variation diagrams of some major and trace elements for the Xiaojiang-Liangnong granitoids and adjacent plutons, and their enclaves. Symbols are same as in Fig. 5. Note that a decreasing $\mathrm{P}_{2} \mathrm{O}_{5}$ with the increasing $\mathrm{SiO}_{2}$ is not consistent with fractionation of crystal in the I-type melt (Chappell and White 1992). 
(a)

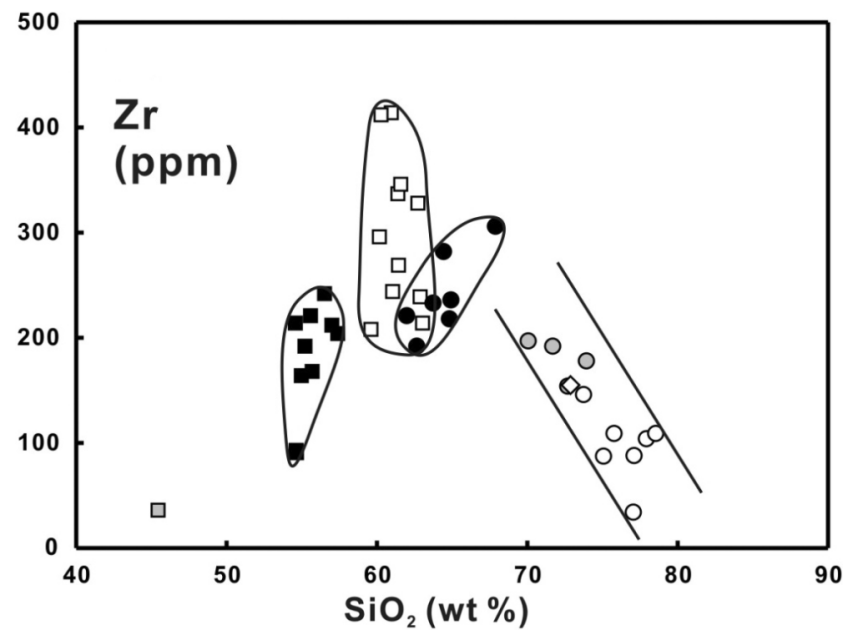

(b)

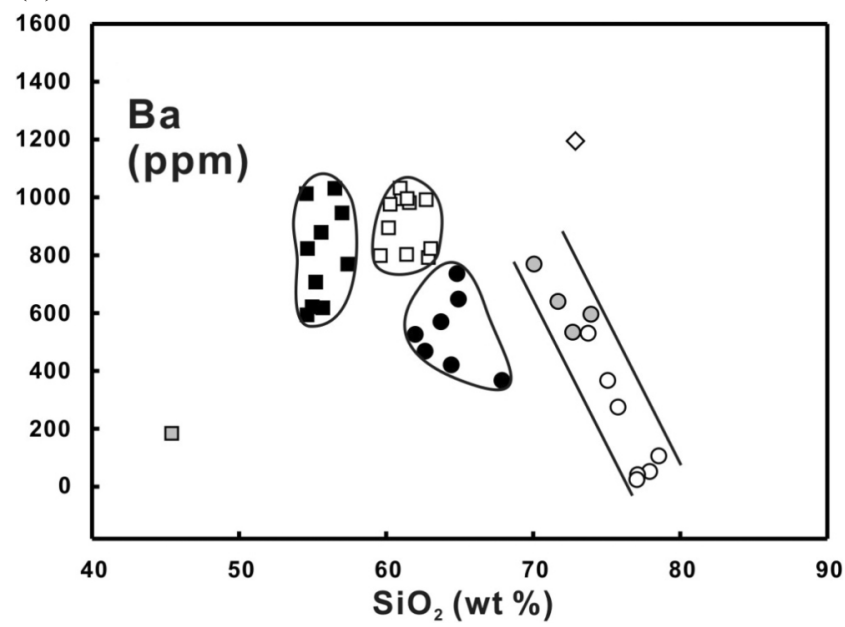

(c)

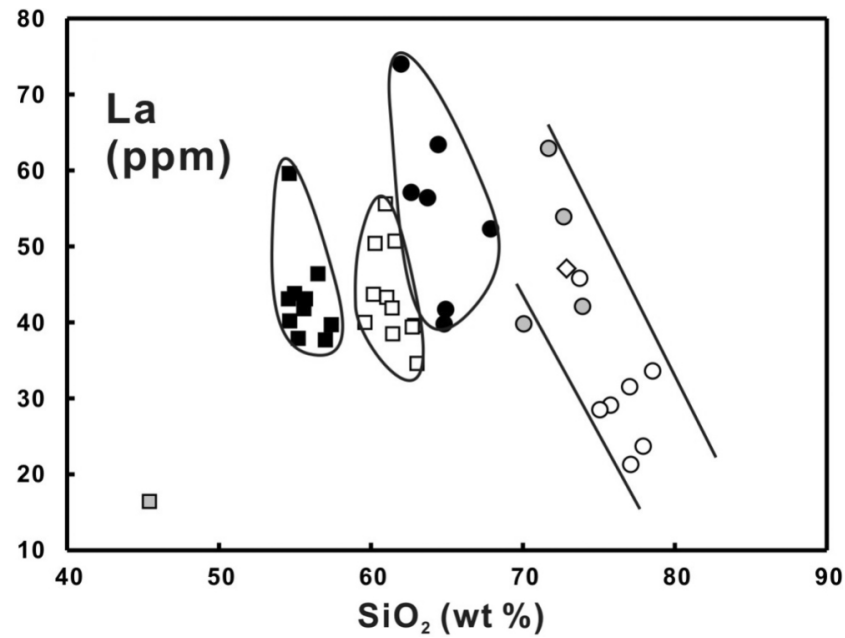

Fig. 7. $\mathrm{Zr}, \mathrm{Ba}$, and $\mathrm{La}$ vs. $\mathrm{SiO}_{2}$ diagrams for the Xiaojiang-Liangnong granitoids and adjacent plutons, and their enclaves. Symbols are same as in Fig. 5. Although there is a general negative correlation between these elements and $\mathrm{SiO}_{2}$ for the felsic rocks $\left(\mathrm{SiO}_{2}>70 \mathrm{wt} \%\right)$, the lack of an obvious positive correlation for the more mafic rocks $\left(\mathrm{SiO}_{2}<\right.$ $70 \mathrm{wt} \%$ ) to cause an inflection (Chappell et al. 1998) for the Xiaojaing-Liangnong granitoids precludes the possibility of a high temperature suite.

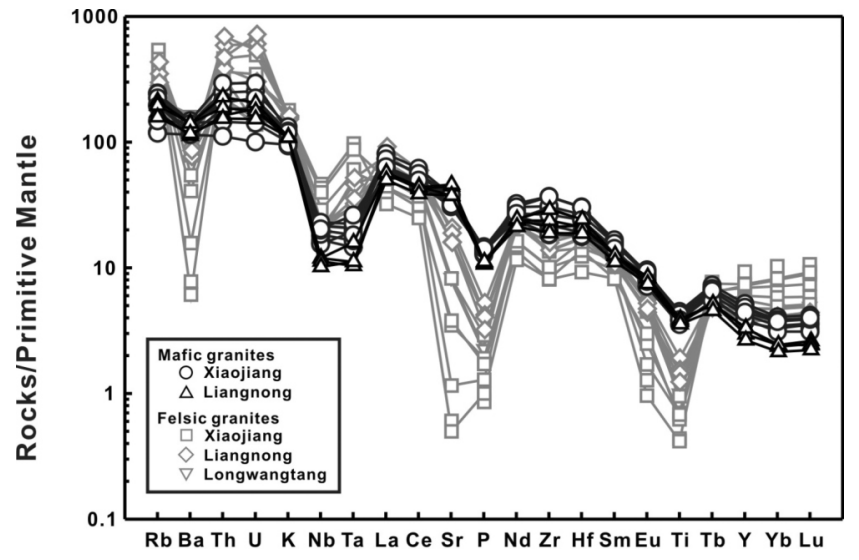

Fig. 8. Primitive mantle-normalized multi-element variation diagrams for mafic and felsic granites of the Xiaojiang-Liangnong igneous complexes. The normalizing values are from McDonough and Sun (1995).

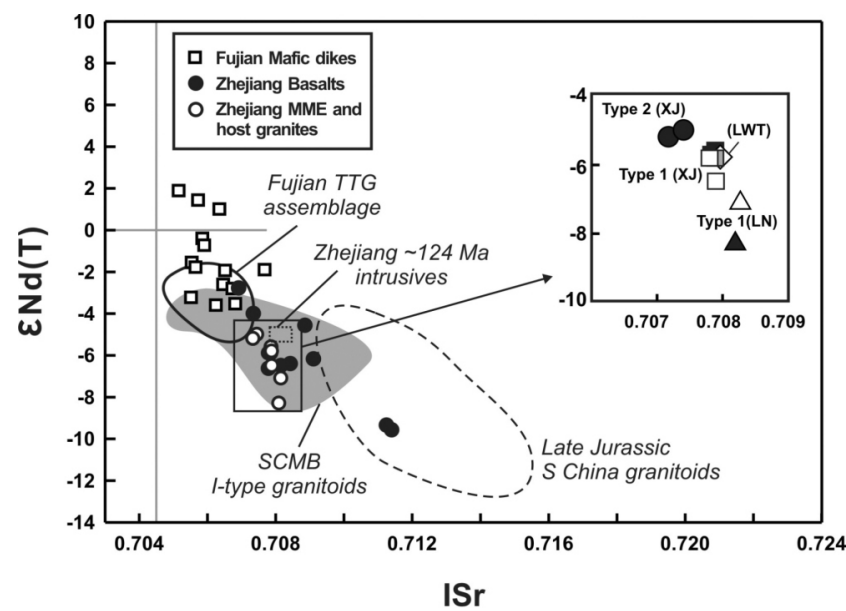

Fig. 9. Sr-Nd isotopic variations for the Xiaojiang-Liangnong granitoids and their enclaves as compared with other Cretaceous granitoids in the SCMB (Lapierre et al. 1997; Chen et al. 2004), Late Jurassic S China granitoids (Hsieh et al. 2008) and Fujian mafic dikes and Zhejiang basalts (our unpublished data). TTG: Trondhjemite-tonalitegranodiorite; XJ: Xiaojiang complex; LN: Liangnong complex; LWT: Longwangtang complex.

cons from Type $2 \mathrm{MME}$ yield one single age in spite a larger number of samples having been conducted (Fig. 4b). These are the zircons without inherited cores. Although the behaviors of $\mathrm{Zr}, \mathrm{Ba}$ and $\mathrm{La}$ show an inflection at $69 \mathrm{wt} \% \mathrm{SiO}_{2}$ (Figs. $7 \mathrm{a}-\mathrm{c}$ ), indicating that these granitic rocks as a whole may be high temperature I-type granites of Chappell's classification scheme, however, constraints set by mineral chemistry, particularly biotites, reveal that only the granodiorite could be of the high-temperature category, and the alkali feldspar granite is uncertain by holding low-Ti biotites.

A direct assessment of the temperature for granitic magmas is the use of zircon saturation temperature (Miller et al. 2003). This is made by comparing the $\mathrm{Zr}$ contents between 
the zircon rims and the host rock under the presence of inherited zircons to ensure saturation in $\mathrm{Zr}$, and the use of the whole-rock bulk composition (Watson and Harrison 1983). Accordingly, $\mathrm{T}_{\mathrm{Zr}}$ calculated for the Xiaojiang granodiorite and Longwangtang alkali feldspar granite are $790-785^{\circ} \mathrm{C}$ (mean $787^{\circ} \mathrm{C}$ ) and $767-761^{\circ} \mathrm{C}$ (mean $763^{\circ} \mathrm{C}$ ), respectively. As for the Type 2 MMEs sample, the temperature is not available because the parameter for the whole-rock bulk composition is away from the recommended value of this thermometry. These results reveal that, although the more mafic granodiorite was emplaced with a higher temperature than the felsic alkali feldspar granite, they are more akin to the low temperature granites, which have a mean $\mathrm{T}_{\mathrm{Zr}}$ of $766^{\circ} \mathrm{C}$ (Miller et al. 2003).

Based on the $\mathrm{U}-\mathrm{Pb}$ zircon and ${ }^{40} \mathrm{Ar} /{ }^{39} \mathrm{Ar}$ biotite age data, the high temperature thermal history of the XiaojiangLiangnong igneous complexes can be estimated. Magmas of granodiorite with a temperature of $790^{\circ} \mathrm{C}\left(\mathrm{T}_{\mathrm{Zr}}\right)$ emplaced at $110 \mathrm{Ma}$ and cooled to $300^{\circ} \mathrm{C}$ (the blocking temperature of biotite; Williams 2001) at $101 \mathrm{Ma}$. Accordingly, a cooling rate of $\sim 54^{\circ} \mathrm{C} \mathrm{myr}^{-1}$ is obtained for the more mafic granitic suite in $\mathrm{N}$ Zhejiang. On the other hand, the cooling rate of felsic granite can be inferred by the Longwangtang pluton. A more rapid rate of $\sim 93^{\circ} \mathrm{C} \mathrm{myr}^{-1}$ is obtained by taking the alkali feldspar granite with a temperature of $767^{\circ} \mathrm{C}\left(\mathrm{T}_{\mathrm{Zr}}\right) \mathrm{em}$ placed at $114 \mathrm{Ma}$ and cooled to $300^{\circ} \mathrm{C}$ at $109 \mathrm{Ma}$. Neighboring plutons can display contrasting cooling rates between the initiation and later stages of one magmatic cycle. For example, the cooling rate of the Changtai pluton (Zhangzhou igneous complex, S Fujian) at temperature higher than $400^{\circ} \mathrm{C}$ is $>300^{\circ} \mathrm{C} \mathrm{Myr}^{-1}$, whereas the adjacent Kunon pluton that intruded at a slightly later time drops down to $40^{\circ} \mathrm{C} \mathrm{Myr}^{-1}$ (Tien et al. 1997). Numerical simulation suggests that extra heat source leading to a high geothermal gradient is necessary to account for a slow cooling rate of $40^{\circ} \mathrm{C} \mathrm{Myr}^{-1}$ in the case of Kunon pluton (Lu et al. 1999). This infers that plutons with slower cooling rates in the thermal regime of the SCMB during 114 - 100 Ma could have been accompanied by basic magma underplating.

\subsection{Origin of MMEs in the Xiaojiang-Liangnong Granitoids}

It is generally accepted that the origin of enclaves in granitoids can be genetically differentiated into four types: xenolithic, restitic, magma segregation, and magma mixing/ mingling (Didier 1991). Basically, the xenolithic and restitic enclaves represent the captured country rocks and residues after melting, respectively, at the time when magma emplaced, and the magma segregation enclaves are cumulates of mafic minerals during the magma evolving stage. The rest, as characterized by the microgranular texture, abundant mafic minerals and specific petrographic features like acicular biotite and apatite, quartz ocelli and poikilitic feldspar are mostly ascribed to the process of magma mixing/mingling. However, the endmember components of magmatic hybridization vary from place to place, and in some cases such MMEs are difficult to distinguish from restites if the granitic magma was formed by partial melting of crustal materials that were composed of pre-existing granitoids (Chappell and White 1992).

The role of MMEs in the petrogenetic model for the generation of granitic magmas is diverse. The general lack of MMEs in mafic granites can be taken to indicate I-type character; and if there is any, a restite origin is favored (Chappell and White 1992). MMEs have also been interpreted as the mantle-derived mafic magma that can mix with a felsic magma originating from the melting of the paragneiss to produce the Hercynian synorogenic monzogranites in the Livradois area of the French Massif Central (Solgadi et al. 2007) and granites in the Nelas area, central Portugal (Silva et al. 2000). On the other hand, MMEs and host granitoids of the central Sierra Nevada batholith (California) are considered as cogenetic, hybrid rocks that were produced by the mixing of the same two components (derived from crustal materials and upper mantle, respectively) in different proportions (Barbarin 2005). Hybridization can occur in both the magma conduits and granitic magma chambers as demonstrated in the Kameruka pluton of the Lachlan Fold Belt (S Australia) (Collins et al. 2000), or in the felsic magma chamber alone as the case in the Miocene Chenar granitoids stock, NW Kerman (Iran) (Arvin et al. 2004).

In the Xiaojiang-Liangnong complexes, two main features are observed between the MMEs and their hosts: (1) mineral compositions of pyroxene, feldspar, biotite, and hornblende are nearly identical (Yen 2005; Figs. 3a-b); and (2) they, including the Type 1a (gabbroic) MMEs, share similarities of the $\mathrm{Sr}$ and $\mathrm{Nd}$ isotopic compositions. On the contrary, dissimilarities also exist. For instance, other than the granitoids, which are all high-K calc alkaline rocks, the Type 2 MMEs fall into the medium-K calc-alkaline field (Fig. 5). Also, judging from the non-linear relationship between Type 2 MMEs and host rocks in Zr, Ba, and La (Fig. 7), these MMEs are more likely to be of exotic origin relative to the alkali feldspar granites. Accordingly, Type 2 MMEs are best explained as the mixing/mingling products of two components, one is the magma of alkali feldspar granite host and the other is the magma that intruded into the alkali feldspar granite at $103 \mathrm{Ma}$ in a form of mafic dike. This is consistent with the fact that basaltic activity has been prevailing during 110 - 97 Ma in N Zhejiang, as exemplified by the bimodal basaltic eruption and mafic dike injection into rhyolites (Lapierre et al. 1997; Chen et al. 2008). The mafic dike is most likely a stream of magma injection from the basic underplating. Referring to the relationship between Type 2 MMEs and host, we suggest that Type 1 MMEs are likewise the products generated from the injection of mafic dike into the granodiorite. Similarly, mixing/mingling between mag- 
mas of mafic dike and granodiorite explains the origin of Type 1 MMEs. The granodioritic body could have been kept in a plastic state under a high geothermal gradient since the time of emplacement, and further heated by the basaltic magmas when Type 1 MMEs were formed. As to the distinct Type 1a gabbroic MMEs, which possess extremely abundant pyroxenes $(>56 \%)$ and more basic geochemical characteristics $\left(\mathrm{SiO}_{2}=45 \mathrm{wt} \%\right)$ than other studied MMEs, we suggest that they represent cumulates crystallized from the intruded basic magma.

Related processes could also have involved fragmentation and partial hybridization of "synplutonic" mafic dikes. The term "synplutonic" denotes that the host pluton has not yet totally solidified at the time of dike injection. This kind of hybridized process has previously been described in the case of the Sierra Nevada batholith (California) by Barbarin (2005). Mafic magma was injected into an open system in which granitoid magma was already moving upward, so that there was not enough time to thoroughly mix with the host and was disrupted into small scattered blobs (e.g., Type 1 MMEs) in the moving granitoid magma. During ascent and emplacement, mingling continued and interaction between granitoids and MMEs involved thermal, mineral, and chemical transfers. Local mixings explain the similar mineralogy and geochemistry of MMEs-granitoid pair. Our Sr and $\mathrm{Nd}$ isotope data further suggest even the possibility of isotopic homogeneity. The similarity of $\mathrm{Sr}$ and $\mathrm{Nd}$ isotope compositions may indicate that the MMEs have been largely modified by the granodiorite host.

Usually, during hybridization, a chilled margin is formed on the hotter basic member leading to a decreasing amount of mafic minerals away from the contact margin (Kadioğlu and Güleç 1999). This phenomenon is simply because mafic dikes had intruded into a low-temperature alkali feldspar granite, which in turn, led to a drastic cooling rate preventing the crystallization of high temperature mafic minerals such as hornblendes in Type 2 MMEs. The large temperature contrast between the injected mafic dike and the host rock is crucial for generating quenching crystals of biotite as there is no such kind of biotite in the Type 1 MMEs. We suggest that hybridization had taken place more vigorously due to the smaller grain size of mineral constituents. The closeness of $\mathrm{Sr}$ and $\mathrm{Nd}$ isotopic compositions of Type 2 MMEs and their hosts is the supportive evidence.

\subsection{Origin of N Zhejiang I-Type Granites and Tectonic Evolution in the SCMB}

When dealing with the origin of Xiaojiang-Liangnong granites, all the Cretaceous granitoids and the tectonic setting that governed the genesis of granitic magmas in the SCMB must be taken into consideration. Three stages of magmatism are recognized for the Late Yanshanian orogeny. The earliest magmatism can be revealed by the 130 - 110 Ma compres- sional stage resulting in the trondhjemite-tonalite-granodiorite assemblage emplaced at $18-24 \mathrm{~km}$. These rocks were interpreted as resulting from partial melting of deepened high-Al gabbro that probably represents relicts of the Jurassic underplate (Chen et al. 2004). On the other hand, A-type rocks in the SCMB, occurred as near-surface granites and rhyolites, appeared in a time span largely concentrated at 94 - $81 \mathrm{Ma}$. They are emplaced and erupted as a consequence of progressive lithospheric extension to $60-70 \%$ of the original thickness (Chen et al. 2008). These two kinds of rock assembly thus represent the syn- and post-collisional environments of the Late Yanshanian orogeny, respectively.

Xiaojiang-Liangnong granites, emplaced during 114 $110 \mathrm{Ma}$, are characterized by shallow intrusions of high-K calc-alkaline I-type granitoids at $4-8 \mathrm{~km}$. Time and depth of magma emplacement of Xiaojiang-Liangnong granites are very similar to the Fuzhou complex in north and Zhangzhou complex in south Fujian. Altogether, they can define a magmatic stage during the LY orogenesis by the different rock association and probably a transitional tectonic environment from syn- to post-collision. Our earlier works, based mainly on the study of Fuzhou and Zhangzhou igneous complexes in Fujian, suggested that the diorite-monzogranite-granite association in the SCMB belongs to I-type granitoids in the extensional tectonic setting (Chen et al. 2000, 2004; Hsieh et al. 2008). In fact, alkali feldspar granites and peralkaline A-type granites are closely related to these I-type granitoids in space, and some alkali feldspar granites were categorized as the aluminous A-type granites (e.g., Qiu et al. 2004). Combining with the geochemical and isotopic compositions of A-type rocks (granites/rhyolites) that postdating these I-type granitoids in this magmatic belt, Chen et al. (2008) postulated that such rocks were derived from partial melting of hydrous, mafic to intermediate rocks in the crust (Roberts and Clemens 1993) as constantly heated by the underplated mafic magma. Nevertheless, mixing between crust- and mantle-derived magmas, as exemplified by the $125-115 \mathrm{Ma}$ Pingtan complex in NE Fujian (Griffin et al. 2002), cannot be totally ruled out. Under this model, basaltic magmas were the major heat supplier, and able to be largely retained in the base of the lower crust or trapped by the highly viscous intermediate to acidic melts in the middle to upper crust.

It seems to be a common feature that I-type granites in the SCMB can be separated into two lines of magmatic evolution based on the biotite compositions. The case of Fuzhou and Zhangzhou igneous complexes in Fujian reveals that their lithologies were largely controlled by crystal fractionation among granodiorite, monzogranite and syenogranite, and alkali feldspar granites, and hence their biotites vary sympathetically between $\mathrm{Ti}$ contents and Fe values in two trends (Fig. 3b). Similarly, the discontinuity of biotite compositions at fixed $\mathrm{Fe}$ values for granitoids in $\mathrm{N}$ Zhejiang is an indication that two host rocks, the granodiorite and the alkali feldspar granite, were derived from different sources. Zircon 
saturation temperatures calculated for these two series of granitic rocks, $787^{\circ} \mathrm{C}$ and $763^{\circ} \mathrm{C}$, respectively, indicate that they are more likely the low temperature I-type granite. This is further supported by the presence of zircon inheritance (Figs. $4 \mathrm{a}, \mathrm{c}$ ) and the lack of associated mineralization (Chappell et al. 1998).

Geochemically, MMEs and hosts show linear trends for various elements on Harker diagrams (Figs. 5, 6, and 7). Chappell (2003a) suggested such linear trends are not consistent with a mechanism of crystal fractionation, and argued that the crystal has been entrained in the melt causing resitite crystal fractionation, with different degrees of mineral separation of felsic melt and mafic residual material producing linear correlations between elements. Only in high temperature conditions, crystals were precipitated from the melt and cumulates were formed. Most notably, $\mathrm{P}_{2} \mathrm{O}_{5}$ increase in abundance with increasing $\mathrm{SiO}_{2}$ is the indication of fractionation of crystal from I-type melts (Chappell and White 1992). The reverse trend of $\mathrm{P}_{2} \mathrm{O}_{5}$ for the studied rocks (Fig. 6e) and the rarity of cumulate rocks in the studied area favor Chappell's idea. The only point that does not fit the concept of low temperature I-type granite is the presence of the $\mathrm{Zr}, \mathrm{Ba}$, and $\mathrm{La}$ inflection pattern. We interpret that the two trends displayed on the different sides of the inflection as representing variation as two kinds of magma evolved separately. The alkali feldspar granite, which appeared earlier, has evolved with a decreasing trend, whereas granodiorite, displays an increasing trend. On this basis, along with the presence of young zircon inheritance, alkali feldspar granite in the Xiaojiang-Liangnong complexes can be explained by melting of quartzofeldspathic sources, whereas granodiorite relates to the melting of mafic source rocks (Chappell et al. 1998), and all these sources are thought to be formed at the LY syncollisional stage.

\section{CONCLUSION}

$\mathrm{U}-\mathrm{Pb}$ zircon ages provide important information for the petrogenetic model for the mafic and felsic granites of the Xiaojiang-Liangnong complexes. Both the mafic and felsic granites show two stages of U-Pb zircon age, 117 and $110 \mathrm{Ma}$ for the former and 120 and $114 \mathrm{Ma}$ for the latter. Zircon saturation temperatures are calculated as $787^{\circ} \mathrm{C}$ and $763^{\circ} \mathrm{C}$ for mafic and felsic granites, respectively, conforming the presence of high-Ti and low-Ti biotites in these two rock types. This implies that magmas of these I-type granites may be generated by partial melting of preexisting granitic crusts that composed of 120 - 117 Ma granitoids, in which the felsic granites were derived from more quartzofeldspathic and the mafic granites, from more dioritic sources. The lower melting temperature of a quartzofeldspathic source can account for the earlier formation of felsic granite (114 Ma) than the mafic granites $(110 \mathrm{Ma})$ under the increasing thermal state at constant underplating of basic magma.
MMEs are of two groups, all represent mixing/mingling products between the injected dikes and their host granitoids. This is evidenced by the fact that MMEs enclosed in felsic granites (Type 2) display one single U-Pb zircon age of $103 \mathrm{Ma}$, but tinged with a distinct medium-K calc-alkaline character. Earlier intrusion renders a more rapid cooling rate of the felsic granites, as exemplified by the Longwangtang alkali feldspar granite body $\left(\sim 93^{\circ} \mathrm{C} \mathrm{myr}^{-1}\right)$. Injection of mafic dikes into a cooler environment explains the crystallization of low-temperature minerals with quenching texture. Those enclosed in mafic granites (Type 1) may represent mafic dike intrusion at a later time. The host granodioritic body had been kept to be plastic as subjected to a higher geothermal gradient and constantly heated by the intrusion of basic magma in an adjacent area. This is the reason for a slower cooling rate of the Xiaojiang-Liangnong granodiorite $\left(\sim 54^{\circ} \mathrm{C} \mathrm{myr}^{-1}\right)$. The common existence of inherited zircon cores in these granitoids support that they were derived from partial melting of preexisting granitic crusts. Earlier emplacement of felsic magmas is the indication of a lower melting point of quartzofeldspathic sources in a thermal regime. This scenario, similar to the recent genetic model for I-type mafic and felsic granites in the LFB, is proposed to record the onset of the post-orogenic stage of the Late Yanshanian movement.

Acknowledgements The authors are thankful to Dr. S. L. Chung for arranging the trace element measurement and zircon U-Pb dating; Dr. H. J. Yang for the Sr-Nd isotope analysis; and Dr. Y. Iizuka for his assistance on zircon CL images. Drs. J. L. Shen and G. F. Xing are appreciated for their assistance in fieldwork and zircon separation. We are grateful to Dr. Mohsen Arvin and an anonymous reviewer for constructive comments. This work was supported by the research grants from the National Science Council, ROC (NSC84, 85-2111-M002-001, 030 and NSC86-2116-M002003/Chen).

\section{REFERENCES}

Andersen, T., 2002: Correction of common lead in U-Pb analyses that do not report ${ }^{204} \mathrm{~Pb}$. Chem. Geol., 192, 59-79, doi: 10.1016/S0009-2541(02)00195-X. [Link]

Arvin, M., S. Dargahi, and A. A. Babaei, 2004: Mafic microgranular enclave swarms in the Chenar granitoids stock, NW of Kerman, Iran: Evidence for magma mingling. $J$. Asian Earth Sci., 24, 105-113, doi: 10.1016/j.jseaes.2003. 09.004. [Link]

Barbarin, B., 2005: Mafic magmatic enclaves and mafic rocks associated with some granitoids of the central Sierra Nevada batholith, California: Nature, origin, and relations with the hosts. Lithos, 80, 155-177, doi: 10.1016/j.lithos. 2004.05.010. [Link]

BGMRZJ (Bureau of Geology and Mineral Resources of Zhejiang Province), 1989: Regional geology of Zhejiang Pro- 
vince. Geological Publishing House, Beijing, 688 pp. (in Chinese)

Cawood, P. A., A. A. Nemchin, M. Freeman, and K. Sircombe, 2003: Linking source and sedimentary basin: Detrital zircon record of sediment flux along a modern river system and implications for provenance studies. Earth Planet. Sci. Lett., 210, 259-268, doi: 10.1016/S0012-821X(03)00122-5. [Link]

Chappell, B. M., 2003a: Towards a unified model of granite petrogenesis. In: Blevin, P., M. Jones, and B. Chappell (Eds.), Magmas to Mineralization: The Ishihara Symposium: Granites and Associated Metallogenesis, 37-38.

Chappell, B. M., 2003b: Causes of variation in granite suites. In: Blevin, P., M. Jones, and B. Chappell (Eds.), Magmas to Mineralization: The Ishihara Symposium: Granites and Associated Metallogenesis, 27-34.

Chappell, B. W., 1999: Aluminium saturation in I- and S-type granites and the characterization of fractionaed haplogranites. Lithos, 46, 535-551 doi: 10.1016/S0024-4937(98) 00086-3. [Link]

Chappell, B. W. and A. J. R. White, 1992: I- and S-type granites in the Lachlan Fold Belt. Trans. R. Soc. Edinburg: Earth Sci., 83, 1-26.

Chappell, B. W., A. J. R. White, and D. Wyborn, 1987: The importance of residual source material (restite) in granite petrogenesis. J. Petrol., 28, 1111-1138.

Chappell, B. W., C. J. Bryant, D. Wyborn, A. J. R. White, and I. S. Williams, 1998: High- and low-temperature I-type granites. Resour. Geol., 48, 225-235, doi: 10.1111/j.17513928.1998.tb00020.x. [Link]

Chen, C. H. and T. C. Tung, 1984: On-line data reduction for electron microprobe analysis. Acta Geol. Taiwan., 22, 196-200.

Chen, C. H., W. Lin, H. Y. Lu, C. Y. Lee, J. L. Tien, and Y. H. Lai, 2000: Cretaceous fractionated I-type granitoids and metaluminous A-type granites in SE China: The Late Yanshanian post-orogenic magmatism. Trans. R. Soc. Edinburgh: Earth Sci., 91, 195-205.

Chen, C. H., W. Lin, C. Y. Lan, and C. Y. Lee, 2004: Geochemical, $\mathrm{Sr}$ and $\mathrm{Nd}$ isotopic characteristics and tectonic implications for three stages of igneous rock in the Late Yanshanian (Cretaceous) orogeny, SE China. Trans. $R$. Soc. Edinburgh: Earth Sci., 95, 237-248, doi: 10.1017/ S0263593300001048. [Link]

Chen, C. H., C. Y. Lee, H. Y. Lu, and P. S. Hsieh, 2008: Generation of Late Cretaceous silicic rocks in SE China: Age, major element and numerical simulation constraints. J. Asian Earth Sci., 31, 479-498, doi: 10.1016/j.jseaes.2007.08.002. [Link]

Chen, J., T. Zhou, C. Yin, and F. A. Foland, 1991: ${ }^{40} \mathrm{Ar} /{ }^{39} \mathrm{Ar}$ dating of several Mesozoic plutons in southeastern Zhejiang Province. Acta Petrol. Sinica, 3, 37-44. (in Chinese)

Chen, J. F. and B. M. Jahn, 1998: Crustal evolution of southeastern China: $\mathrm{Nd}$ and $\mathrm{Sr}$ isotopic evidence. Tectonophysics, 284, 101-133, doi: 10.1016/S0040-1951(97)00186-8. [Link]
Chen, R., G. F. Xing, Z. L. Yang, J. L. Shen, and Y. Z. Zhou, 2005: Study on Beizhang and Liangnong granites and their dark enclaves. Geol. J. China Univ., 11, 264-275. (in Chinese)

Chiu, H. I., S. L. Chung, F. Y. Wu, D. Liu, Y. H. Liang, I. J. Lin, Y. Iizuka, L. W. Xie, Y. Wang, and M. F. Chu, 2009: Zircon $\mathrm{U}-\mathrm{Pb}$ and $\mathrm{Hf}$ isotopic constraints from eastern Transhimalayan batholiths on the precollisional magmatic and tectonic evolution in southern Tibet. Tectonophysics, doi: 10.1016/j.tecto.2009.02.034, in press.

Collins, W. J., S. R. Richards, B. E. Healy, and P. I. Ellison, 2000: Origin of heterogeneous mafic enclaves by twostage hybridisation in magma conduits (dikes) below and in granitic magma chambers. Trans. R. Soc. Edinburg: Earth Sci., 91, 27-45.

Compston, W., I. S. Williams, J. L. Kirschvink, Z. Zichao, and M. A. Guogan, 1992: Zircon U-Pb ages for the Early Cambrian time-scale. J. Geol. Soc. London, 149, 171-184, doi: 10.1144/gsjgs.149.2.0171. [Link]

Didier, J., 1991: The main types of enclaves in the Hercynian granitoids of the Massif Central, France. In: Didier, J. and B. Barbarin (Eds.), Enclaves and Granite Petrology. Elsevier Science Publishers, New York, 47-61.

Griffin, W. L., X. Wang, S. E. Jackson, N. J. Pearson, S. Y. O'Reilly, X. Xu, and X. Zhou, 2002: Zircon chemistry and magma mixing, SE China: In-situ analysis of Hf isotopes, Tonglu and Pingtan igneous complexes. Lithos, 61, 237 269, doi: 10.1016/S0024-4937(02)00082-8. [Link]

Henry, D. J., C. V. Guidotti, and J. A. Thomson, 2005: The Ti-saturation surface for low-to-medium pressure metapelitic biotites: Implications for geothermometry and Tisubstitution mechanisms. Am. Mineral., 90, 316-328, doi: 10.2138/am.2005.1498. [Link]

Hsieh, P. S., C. H. Chen, H. J. Yang, and C. Y. Lee, 2008: Petrogenesis of the Nanling Mountains granites from South China: Constraints from systematic apatite geochemistry and whole-rock geochemical and $\mathrm{Sr}-\mathrm{Nd}$ isotope compositions. J. Asian Earth Sci., 33, 428-451, doi: 10.1016/j. jseaes.2008.02.002. [Link]

Jackson, S. E., N. J. Pearson, W. L. Griffin, and E. A. Belousova, 2004: The application of laser ablation-inductively coupled plasma-mass spectrometry to in situ U-Pb zircon geochronology. Chem. Geol., 211, 47-69, doi: 10.1016/j. chemgeo.2004.06.017. [Link]

Jahn, B. M., X. H. Zhou, and J. L. Li, 1990: Formation and tectonic evolution of Southeastern China and Taiwan: Isotopic and geochemical constraints. Tectonophysics, 183, 145-160, doi: 10.1016/0040-1951(90)90413-3. [Link]

Kadioğlu, Y. K. and N. Güleç, 1999: Types and genesis of the enclaves in Central Anatolian granitoids. Geol. J., 34, 243-256, doi: 10.1002/(SICI)1099-1034(199907/09)34:3 $<243:: A I D-G J 825>3.0 . C O ; 2-\#$. [Link]

Lai, Y. H., 1995: Geochemistry and mineral chemistry of Cretaceous granites in Fujian, SE China. Master Thesis, Dept. Geology, National Taiwan Univ., 94 pp. (in Chinese) 
Lapierre, H., B. M. Jahn, J. Charvet, and Y. W. Yu, 1997: Mesozoic felsic arc magmatism and continental olivine tholeiites in Zhejiang Province and their relationship with the tectonic activity in southeastern China. Tectonophysics, 274, 321-338, doi: 10.1016/S0040-1951(97)00009-7. [Link]

Leake, B. E., 1978: Nomenclature of amphiboles. Can. Mineral., 16, 501-520.

Lee, C. Y., J. H. Tsai, H. H. Ho, T. F. Yang, S. L. Chung, and C. H. Chen, 1997: Quantitative analysis in rock samples by an X-ray fluorescence spectrometer, (I) major elements (Abs.). Annual Meeting of Geological Society of China, 418-420. (in Chinese)

Lu, H. Y., J. L. Tien, C. H. Lo, and C. H. Chen, 1999: Thermal modeling and chronological constraints of granitic plutonism in Zhangzhou Igneous Complex, SE China. J. Geol. Soc. China, 42, 521-541.

Ludwig, K. R., 2003: ISOPLOT 3: A geochronological toolkit for Microsoft excel. Berkeley Geochrono. Lab. Spec. Publ., 4, $74 \mathrm{pp}$.

Martin, H., B. Bonin, R. Capdevila, B. M. Jahn, J. Lameyre, and Y. Wang, 1994: The Kuiqi peralkaline granitic complex (SE China): Petrology and geochemistry. J. Petrol., 35, 983-1015.

McDonough, W. F. and S. S. Sun, 1995: The composition of the earth. Chem. Geol., 120, 223-253, doi: 10.1016/0009-2541 (94)00140-4. [Link]

Miller, C. F., S. M. McDowell, and R. W. Mapes, 2003: Hot and cold granites? Implications of zircon saturation temperatures and preservation of inheritance. Geology, 31, 529532, doi: 10.1130/0091-7613(2003)031<0529:HACGIO> 2.0.CO;2. [Link]

Pitcher, W. S., 1997: The Nature and Origin of Granite. London: Chapman \& Hall, 416 pp.

Qiu, J. S., D. Z. Wang, B. I. A. McInnes, S. Y. Jiang, R. C. Wang, and S. Kanisawa, 2004: Two subgroups of A-type granites in the coastal area of Zhejiang and Fujian Provinces, SE China: Age and geochemical constraints on their petrogenesis. Trans. R. Soc. Edinburgh: Earth Sci., 95, 227-236, doi: 10.1017/S0263593300001036. [Link]

Roberts, M. P. and J. D. Clemens, 1993: Origin of high-potas- sium, calc-alkaline, I-type granitoids. Geology, 21, 825828, doi: 10.1130/0091-7613(1993)021<0825:OOHPTA $>$ 2.3.CO;2. [Link]

Schmidt, M. W., 1992: Amphibole composition in tonalite as a function of pressure: An experimental calibration of the Al-in-hornblende barometer. Contrib. Mineral. Petrol., 110, 304-310, doi: 10.1007/BF00310745. [Link]

Silva, M. M. V. G., A. M. R. Neiva, and M. J. Whitehouse, 2000: Geochemistry of enclaves and host granites from the Nelas area, central Portugal. Lithos, 50, 153-170, doi: 10.1016/S0024-4937(99)00053-5. [Link]

Solgadi, F., J. F. Moyen, O. Vanderhaeghe, E. W. Sawyer, and L. Reisberg, 2007: The role of crustal anatexis and mantle-derived magmas in the genesis of synorogenic Hercynian granites of the Livradois area, French Massif Central. Can. Mineral., 45, 581-606, doi: 10.2113/gscanmin. 45.3.581. [Link]

Tien, J. L., C. H Chen, C. H. Lo, and Y. S. Lai, 1997: Contrasting cooling rates of coeval granitic plutons in the Zhangzhou Igneous Complex, SE China: Evidences from ${ }^{40} \mathrm{Ar} /{ }^{39} \mathrm{Ar}$ thermochronology and Al-amphibole geobarometer. $J$. Geol. Soc. China, 40, 607-624.

Watson, E. B. and T. M. Harrison, 1983: Zircon saturation revisited: Temperature and composition effects in a variety of crustal magma types. Earth Planet. Sci. Lett., 64, 295304, doi: 10.1016/0012-821X(83)90211-X. [Link]

Williams, I. S., 1998: U-Th-Pb geochronology by ion microprobe. In: McKibben, M. A., W. C. Shanks III, and W. I. Ridley (Eds.), Applications of Microanalytical Techniques to Understanding Mineralizing Processes, 1-35.

Williams, I. S., 2001: Response of detrital zircon and monazite, and their $\mathrm{U}-\mathrm{Pb}$ isotopic systems, to regional metamorphism and host-rock partial melting, Cooma Complex, southeastern Australia. Aust. J. Earth Sci., 48, 557-580, doi: 10.1046/j.1440-0952.2001.00883.x. [Link]

Yen, C. M., 2005: Mineral chemistry and geochemistry of the Xiaojiang-Liangnong complexes and their enclaves in Zhejiang. Master Thesis, Dept. Geosciences, National Taiwan Univ., 138 pp. (in Chinese) 